\title{
Rapid Assembly of Functional Presynaptic Boutons Triggered by Adhesive Contacts
}

\author{
Anna Lisa Lucido, ${ }^{1,4}$ Fernando Suarez Sanchez, ${ }^{2,4}$ Peter Thostrup, ${ }^{2,4}$ Adam V. Kwiatkowski, ${ }^{5}$ Sergio Leal-Ortiz, ${ }^{6}$ \\ Gopakumar Gopalakrishnan, ${ }^{1,3,4}$ Dalinda Liazoghli, ${ }^{1,4}$ Wiam Belkaid, ${ }^{1,4}$ R. Bruce Lennox, ${ }^{3,4}$ Peter Grutter, ${ }^{2,4}$ \\ Craig C. Garner, ${ }^{6}$ and David R. Colman ${ }^{1,4}$ \\ ${ }^{1}$ Montreal Neurological Institute and Hospital, McGill University, Montreal, Quebec H3A 2B4, Canada, ${ }^{2}$ Department of Physics, McGill University, \\ Montreal, Quebec H3A 2T8, Canada, ${ }^{3}$ Department of Chemistry, McGill University, Montreal, Quebec H3A 2K6, Canada ${ }^{4}$ Program in NeuroEngineering, \\ McGill University, Montreal, Quebec H3A 2B4, Canada, ${ }^{5}$ Department of Biological Sciences, Stanford University, Palo Alto, California 94305 , and \\ ${ }^{6}$ Department of Psychiatry and Behavioral Science, Nancy Pritzker Laboratory, Stanford University, Palo Alto, California 94304
}

CNS synapse assembly typically follows after stable contacts between "appropriate" axonal and dendritic membranes are made. We show that presynaptic boutons selectively form de novo following neuronal fiber adhesion to beads coated with poly-D-lysine (PDL), an artificial cationic polypeptide. As demonstrated by atomic force and live confocal microscopy, functional presynaptic boutons self-assemble as rapidly as $1 \mathrm{~h}$ after bead contact, and are found to contain a variety of proteins characteristic of presynaptic endings. Interestingly, presynaptic compartment assembly does not depend on the presence of a biological postsynaptic membrane surface. Rather, heparan sulfate proteoglycans, including syndecan-2, as well as others possibly adsorbed onto the bead matrix or expressed on the axon surface, are required for assembly to proceed by a mechanism dependent on the dynamic reorganization of F-actin. Our results indicate that certain (but not all) nonspecific cationic molecules like PDL, with presumably electrostatically mediated adhesive properties, can effectively bypass cognate and natural postsynaptic ligands to trigger presynaptic assembly in the absence of specific target recognition. In contrast, we find that postsynaptic compartment assembly depends on the prior presence of a mature presynaptic ending.

\section{Introduction}

Synapses are asymmetric sites of cell-cell contact that mediate the vectorial transfer of information between neuronal cells and their targets. In recent years, numerous proteins comprising the molecular architecture of the synaptic junction have been identified (Kim and Sheng, 2004; Okabe, 2007; Jin and Garner, 2008). Many of these proteins, particularly those of the presynaptic ending, can assemble rapidly following nascent axodendritic contact (Ahmari et al., 2000; Friedman et al., 2000), and this process can be driven artificially by transmembrane cleft-spanning molecular arrays such as neurexin-neuroligin pairs, NGL-2 and/or SynCAM (Scheiffele et al., 2000; Biederer et al., 2002; Graf et al., 2004; Kim et al., 2006). Eventually, the synaptic junctional complex becomes remarkably resistant to degradation such that only membrane-disruptive treatments

\footnotetext{
Received March 19, 2009; revised July 24, 2009; accepted Aug. 4, 2009.

This work was supported by a grant from the Canadian Institutes for Health Research and funds from Rio Tinto Alcan and the Molson Foundation (D.R.C.), and by the National Institutes of Health (NS39471, NS053862, and NS353862 to C.C.G.). A.L.L. was supported by a doctoral training award from the Fonds de la Recherche en Santé de Québec. F.S.S. was supported by a National Council for Science and Technology Fellowship. A.V.K. was supported by National Research Service Award Grant 5T32 CA09302. The monoclonal antibody anti- $\beta$-tubulin developed by M. Klymkowsky was obtained from the Developmental Studies Hybridoma Bank developed under the auspices of the National Institute of Child Health and Human Development and maintained by the Department of Biological Sciences, The University of lowa (lowa City, IA). We thank Mariette Lavallée and Dr. Neil Cameron for technical support as well as Drs. Edward Ruthazer, Elise Stanley, and Alexander C. Jackson for helpful comments and discussions.

Correspondence should be addressed to Dr. David R. Colman, Montreal Neurological Institute, McGill University, 3801 University Street, Room 636, Montreal, QC H3A 2B4, Canada. E-mail: david.colman@mcgill.ca. DOI:10.1523/JNEUROSCI.1381-09.2009

Copyright $\odot 2009$ Society for Neuroscience $\quad 0270-6474 / 09 / 2912449-18 \$ 15.00 / 0$
}

will lead to dissolution of the complex (Phillips et al., 2001). Collectively, these data reveal that constituents of the synapse can integrate, dissociate and reassemble quite readily, and disclose properties that would likely be essential features for synapse formation, pruning, and re-formation in situ. Of course, many molecular details of synaptogenesis are lacking, due to the rarity of observing these rapid events in vitro and in vivo and the difficulty in temporally controlling nascent synapse formation during neuronal development.

In this study, we examined CNS synapse formation using an artificial matrix to induce the assembly of a presynaptic ending that appears strikingly similar to one produced in situ. The paradigm is based on earlier observations showing that cultured cerebellar neurons form presynaptic-like specializations when placed in contact with beads coated with positively charged proteins including poly-L-lysine (PLL) and argininerich histones, but not with beads coated with negative or neutral charge (Burry, 1980, 1982; Peng et al., 1987). These studies imply that target adhesion alone can trigger presynaptic endings to form in the absence of any specialized cues. However, it was never clear from these early studies whether these synaptic vesicle-filled varicosities were functional, whether the requirements for their formation were intrinsic to axons or dependent on dendritic contact, or whether the assembly process was similar to that observed in situ.

Using atomic force microscopy (AFM), we first show that poly-D-lysine (PDL)-coated beads (PDL beads) induce adhesion to axonal processes such that the bead is rapidly resistant to de- 
tachment within minutes of contact. Our data reveal that PDL beads can indeed induce the formation of presynaptic boutons by a mechanism that depends on F-actin reorganization and the presence of heparan sulfate proteoglycans (HSPGs), and implicate the cell surface HSPG syndecan-2 as at least one important coreceptor. Functional presynaptic differentiation is fast, on a time scale of minutes, similar to native synapse formation and can occur in the absence of postsynaptic partners, while postsynaptic assembly is dependent on the presence of differentiated presynaptic structures. These findings suggest that early recognition events that guide synaptic assembly need not be highly selective on axons, and reveal a readiness for axons to form noncognate presynaptic connections at any site along their length. Together, these data strengthen previous conclusions that the presynaptic complex is primed for self-assembly once presented with appropriate triggering signals in a permissible environment (Burry, 1982; Phillips et al., 2001).

\section{Materials and Methods}

Neuronal culture. All animal experimentation was approved by the institutional animal care committee and conformed to the guidelines of the Canadian Council of Animal Care. All culture media was purchased from Invitrogen. Hippocampi were dissected from embryonic day 17-18 (E17-E18) Sprague Dawley rat embryos (Charles River) as described previously (Benson and Tanaka, 1998). For immunocytochemistry, cells were plated at a density of $2.0-2.5 \times 10^{4} \mathrm{~cm}^{-1}$ on PDL (Sigma-Aldrich)coated glass coverslips. For electron microscopy, cells were plated on PDL-treated four-well plates (Nunc, VWR) at a density of $5.0 \times 10^{4}$ cells per well. All cells were cultured in serum-free Neurobasal medium supplemented with L-glutamine and B-27. Transfection of plasmid DNA was performed using Lipofectamine (Invitrogen) according to the company protocol, and transfected cells were incubated for a minimum of $48 \mathrm{~h}$ before experimentation. Mouse synaptophysin-EGFP plasmid was a gift from Dr. Edward Ruthazer (McGill University), and pEGFP-C1 plasmid was purchased from Clontech.

Lentiviral-infected hippocampal cultures were prepared using a modified Banker-style protocol (Banker and Goslin, 1998) and infected as described previously (Leal-Ortiz et al., 2008). Three constructs were used for these studies. These included EGFP-tagged versions of SV2, $\alpha$-SAP97, and an mCherry-tagged version of $\beta$-actin. In the case of SV2 and $\alpha$-SAP97, the EGFP-fusion protein was expressed under the ubiquitin promoter with the FUGW lentiviral vector as previously described (Schlüter et al., 2006; Leal-Ortiz et al., 2008; Waites et al., 2009). With regard to the mCherry- $\beta$ Actin construct, the CMV-enhancer/chicken $\beta$-actin/ $\beta$-globin intron (CAG) promoter driving transgene expression in the lentiviral expression vector pLL4.4 (Kwiatkowski et al., 2007; Kwiatkowski et al., 2009) was replaced with a ubiquitin promoter, creating pLL4.5. The EGFP expression cassette in pLL4.5 was excised and replaced with a fusion between mCherry and human $\beta$-actin (gift from Frank Gertler, Massachusetts Institute of Technology, Cambridge, MA), creating pLL4.5 mCherry- $\beta$-actin.

Preparation of micropatterned glass substrates. Glass substrates were patterned with polylysine using custom-made silicone elastomer stamps. To facilitate transfer of polylysine to the glass, coverslips were first surface-treated with 1\% 3-glycidoxypropyl-trimethoxysilane (3-GPS, Gelest) in toluene for $1 \mathrm{~h}$, transferred to a beaker containing fresh toluene, and rinsed several times. Coverslips were then dried with nitrogen steam and autoclaved.

Polydimethylsiloxane (PDMS) was prepared from the Sylgard 184 Silicone elastomer kit (Dow Corning) according to company protocols and poured onto a master silicone wafer etched with the following grid-like dimensions: $10-\mu \mathrm{m}$-wide lines spaced $420 \mu \mathrm{m}$ apart with $20 \mu \mathrm{m}$ circles centered on line intersections. The PDMS was left standing on the wafer for $1 \mathrm{~h}$ at room temperature followed by curing at $60^{\circ} \mathrm{C}$ for $12 \mathrm{~h}$, then carefully peeled off of the wafer.

For stamping coverslips, the molded PDMS stamp was sterilized under UV light, soaked in $70 \%$ ethanol for $1 \mathrm{~min}$, and then dried using nitrogen gas. The stamp was placed in a solution containing poly-D-lysine (1 $\mathrm{mg} / \mathrm{ml}$ in PBS, $\mathrm{pH}$ 7.4) for $3 \mathrm{~h}$, dried with nitrogen gas, and then applied to the treated coverslips with gentle pressure for several seconds. The coverslips were then rinsed several times with sterile water, placed into culture dishes and used for plating neurons as described above. Cultures grown on these substrates were incubated with poly-D-lysine-coated beads after $7 \mathrm{~d}$ in vitro (DIV).

Preparation of polylysine-coated beads. Neurons were cultured to various stages of development (between DIV7 and DIV21) before the addition of beads. For coating with PDL or poly-L-lysine-FITC (PLL-FITC) (both Sigma), $7 \mu \mathrm{m}$ polystyrene beads (Bangs Laboratories) were incubated with a solution of either polymer in sterile PBS $(50 \mu \mathrm{g} / \mathrm{ml}$ unless otherwise stated), overnight at $4^{\circ} \mathrm{C}$ with end-to-end mixing. Beads were then washed three times in PBS by centrifugation, resuspended in Neurobasal medium, and added dropwise to the neurons at a concentration of $10^{5}$ to $1.5 \times 10^{5}$ beads/coverslip. Uncoated beads were washed in PBS alone and added to the neurons at a density three to four times that of PDL-coated beads to account for the increased proportion of beads that did not adhere to the cultures.

Preparation of lipid bilayer-coated beads. Silica beads (5 $\mu \mathrm{m}$; Bangs Laboratories) were diluted to a concentration of $9,000,000$ beads $/ \mathrm{ml}$ in PBS, washed twice in PBS by centrifugation, and then resuspended and incubated in $1 \mathrm{ml}$ of PBS containing $0.05 \mathrm{mg} / \mathrm{ml}$ avidin overnight at $4^{\circ} \mathrm{C}$. The avidin-treated beads were then washed several times and resuspended in a final volume of $500 \mu \mathrm{l}$ of PBS before incubation with the lipids.

The following lipids purchased from Avanti Polar Lipids were used for the preparation of the bilayer membrane: 1,2-dioleoyl-sn-glycero-3phosphatidylcholine (DOPC), 1,2-dioleoyl-3-trimethylammonium-propane (chloride salt) (DOTAP), and 1,2-distearoyl-sn-glycero-3-phosphatidylethanolamine- $N$-[biotinyl (polyethyleneglycol)2000] (ammonium salt) (DSPE-PEG2000-biotin). Chloroform solutions of DOPC (1.5 mM, 75 $\mu \mathrm{l})$, DOTAP $(1.5 \mathrm{~mm}, 25 \mu \mathrm{l})$, and DSPE-PEG2000-biotin $(0.6 \mathrm{~mm}, 5 \mu \mathrm{l})$ were mixed and dried in vacuum for $4 \mathrm{~h}$ under sterile conditions. The film was then hydrated using $0.1 \mathrm{~m}$ sucrose through rapid mixing followed by sonication in a bath sonicator for $5 \mathrm{~min}$, which results in the formation of small unilamellar vesicles.

A $500 \mu \mathrm{l}$ quantity of the vesicle solution was then mixed with $500 \mu \mathrm{l}$ of the avidin-coated silica beads, shaken gently, and incubated for $10 \mathrm{~min}$. The bead-vesicle solution was then mixed vigorously and sonicated for 1 min followed by centrifugation (12,000 rpm for $13 \mathrm{~min}$ ), and the resulting pellet was then resuspended in PBS. The bilayer-coated beads were used within 1 week of preparation and added to cultures as described above.

Drug treatments. In cultures to which inhibitors of HSPGs were added, heparinase (III or II), heparan sulfate (all Sigma), or heparin (Organon) were added immediately before the addition of PDL-coated beads. Heparinase was diluted in Neurobasal medium and added to cells for a final concentration of 1-1.25 Sigma units/ml, while heparan sulfate or heparin was diluted to concentration of either $1 \mu \mathrm{g} / \mathrm{ml}$ or $20 \mu \mathrm{g} / \mathrm{ml}$. In cultures where inhibitors of actin were added, jasplakinolide (Invitrogen) and latrunculin A (Sigma) were diluted from a 1000× stock in DMSO (Invitrogen) to a final concentration of $5 \mu \mathrm{M}$ in Neurobasal medium and added to DIV13-DIV15 cultures immediately before the addition of PDL-coated beads.

Immunocytochemistry. Cells were fixed with $4 \%$ paraformaldehyde (Sigma) in phosphate buffer (PB), pH 7.4, for $25 \mathrm{~min}$, incubated in blocking solution [Tris-buffered saline, $\mathrm{pH} 7.4$ (TBS), containing 4\% normal donkey serum and $0.1 \%$ Triton X-100] for $30 \mathrm{~min}$, followed by incubation in primary antibodies diluted in TBS containing $0.1 \%$ Triton $\mathrm{X}-100$ and $0.5 \%$ normal donkey serum (NDS, Jackson ImmunoResearch), overnight at $4^{\circ} \mathrm{C}$ with gentle rotation. Cells were washed in TBS, incubated in fluorochrome-coupled secondary antibodies (in TBS- $0.5 \%$ NDS), washed three times in TBS, and mounted on glass slides. Primary antibodies used for our studies include rabbit polyclonal anti-Synaptophysin (Invitrogen), mouse monoclonal anti-Bassoon (Assay Designs), rabbit polyclonal anti-CaV2.2 (Ab571, gift from Dr. E. Stanley, University of Toronto, Toronto, ON, Canada), mouse monoclonal anti-RIM (BD Biosciences), guinea pig polyclonal VGlut1 (Millipore Bioscience Research Reagents), rabbit polyclonal anti-glutamate decarboxylase 65/67 
(GAD65/67) (Millipore Bioscience Research Reagents), mouse monoclonal anti-PSD95 (Affinity Bioreagents), rabbit polyclonal anti-N-cadherin (Fannon and Colman, 1996), mouse monoclonal anti-Tau1 (Millipore Bioscience Research Reagents), chicken polyclonal anti-MAP2 (GeneTex), goat polyclonal anti-syndecan-2 (Santa Cruz Biotechnology), mouse monoclonal anti-heparan sulfate 10E4 (Seikagaku), and mouse monoclonal anti- $\beta$-Tubulin (Developmental Studies Hybridoma Bank, see acknowledgments). All secondary antibodies (species-specific, highly cross-adsorbed IgG) were purchased from either Jackson ImmunoResearch or Invitrogen and used at a dilution of 1:200 to 1:500. For cultures that were probed for actin labeling, we added Alexa 488-phalloidin (Invitrogen) to the secondary antibody solution at a concentration of 1:50.

Confocal microscopy of antibody-labeled cultures. Fixed coverslips were imaged using an Olympus Fluoview FV1000 laser scanning confocal microscope with a $60 \times$ PlanApo oil-immersion objective [1.4 numerical aperture (NA)] on an IX81 inverted microscope. Images of single optical sections through the neuritic plane were acquired with $1 \times$ digital zoom and $4 \times$ Kalman averaging. At least 10 separate images were taken for each condition. For double or triple immunostaining, images were acquired via sequential scanning of each individual channel along with the corresponding brightfield (differential interference contrast, or DIC image). For each coverslip, optimal parameters were adjusted manually to avoid image saturation.

Electron microscopy. Unless otherwise stated, all electron microscopy (EM) reagents purchased from Cedarlane. Cells were grown directly on tissue culture plastic (PDL-coated four-well plates, Nunc) to DIV14DIV15, and following incubation with beads for $24 \mathrm{~h}$ were fixed with $2 \%$ glutaraldehyde-2\% PFA (Sigma) for $30 \mathrm{~min}$. For transmission electron microscopy (TEM), cells were then postfixed with $1-2 \% \mathrm{OsO}_{4}$, dehydrated in graded alcohols, embedded in epon, ultrathin-sectioned ( 80 $\mathrm{nm}$ thick), counterstained with uranyl acetate and lead citrate, and examined using a JEOL 100CX transmission electron microscope set to 80 $\mathrm{kV}$ voltage. For scanning electron microscopy, cells were gently removed from their wells via gentle scraping and placed directly on copper grids, dried, and examined using a Hitachi S-4700 Field Emission scanning electron microscope set between 1 and $3 \mathrm{kV}$ voltage.

Combined fluorescence and atomic force microscopy. For these experiments, hippocampal neurons were cultured in $35 \mathrm{~mm}$ glass-bottom dishes (MatTek) coated with PDL. Cells were transfected with mouse synaptophysin-EGFP at DIV6 and incubated for 2-4 d before experimentation. Polystyrene beads $(7 \mu \mathrm{m})$ were attached to an AFM cantilever tip (Veeco, model: MSCT-AUHW) with a UV-curable adhesive (Electrolite). The adhesive was applied to the cantilever tip using a pulled glass capillary (World Precision Instruments) mounted on a micromanipulator stage (MX7600R, Syskiyou). Beads were then picked up with the glued cantilever (mounted in the AFM) from a microscope slide. For the PDL-coated bead experiments, the beaded cantilever was then incubated in $50 \mu \mathrm{g} / \mathrm{ml} \mathrm{PDL}$ solution overnight at $4^{\circ} \mathrm{C}$.

Simultaneous adhesion and live imaging experiments were performed on a Bioscope AFM (Digital Instruments, Veeco Metrology Group), using a $100 \times$ objective (1.45 NA) and Cascade:1k CCD Camera (Photometrics). Cells were mounted onto a heated stage (Warner Instruments) and kept at $37^{\circ} \mathrm{C}$ for the duration of the experiments ( 2 to $3 \mathrm{~h}$ ). Here the AFM function was used to position and move the beaded cantilever with submicrometer precision.

Live imaging experiments using lentiviral-infected neurons. These experiments were performed on a custom-built imaging system comprised of a spinning disc confocal head (Perkin-Elmer), a Zeiss Axiovert 200M microscope, an argon/krypton laser (SpectraPhysics) driven by MetaMorph software (Molecular Devices). Neuronal coverslips were mounted in a custom-built chamber designed for closed perfusion, heated to $37^{\circ} \mathrm{C}$ by forced-air blower. Images were collected with a Zeiss $63 \times$ PlanNeofluar oil-immersion objective (NA 1.4) and a Hamamatsu 512B CCD camera using FITC and Texas Red filter sets (Chroma).

For the time-lapse imaging experiments, cells were mounted onto the microscope stage and perfused with a specially prepared medium (Air medium) containing 25 mM HEPES, B-27, GlutaMAX, penicillin/streptomycin, $5 \mathrm{~mm}$ D-glucose, and $25 \mu \mathrm{M} \beta$-mercaptoethanol in a 1:1 mixture of L-15 and
Hanks' balanced solutions. D-Glucose and $\beta$-mercaptoethanol are from Sigma and all other reagents are from Invitrogen.

Frames were acquired sequentially with laser intensity kept low to avoid photobleaching and laser-induced toxicity. For each movie, several frames were acquired before the addition of PDL beads, which were added thereafter by resuspension in Air medium and perfusion over the cells. The beads were allowed to settle and then time-lapse imaging was quickly resumed within 3 min of their addition. The frames within an imaged field were acquired as $Z$-stacks $(0.5 \mu \mathrm{m}$ width, 7 frames per field $)$ at $30 \mathrm{~s}$ time intervals for the first hour, followed by variable intervals of 1-3 min for longer movies.

Styryl FM4-64 dye imaging. For experiments using noninfected cultures, cells were plated on $42 \mathrm{~mm}$ glass coverslips (Hemogenix) at a density of 400,000/coverslip and cultured for 12-15 d before the addition of PDL-coated beads for $24 \mathrm{~h}$. HBSS (Invitrogen) containing $\mathrm{Ca}^{2+}(1.26$ $\mathrm{mm}), \mathrm{Mg}^{2+}(0.9 \mathrm{~mm})$, and D-glucose $(5.6 \mathrm{~mm})$ was used in all experiments. Hyperkalemic solutions were prepared by addition of $\mathrm{KCl}$ for a final $\mathrm{K}^{+}$concentration of $45 \mathrm{~mm}$. For fluorescence imaging, we used a Zeiss LSM 510 META laser scanning confocal microscope with a $63 \times$ PlanNeofluar oil-immersion objective [1.4 numerical aperture] on a Zeiss Axiovert 100M inverted microscope. Laser intensity was set to $25 \%$ of the maximum to avoid photobleaching and toxicity. Imaging parameters were kept constant throughout all experimental sessions.

Coverslips were mounted onto a heated stage, rinsed $1 \times$ with warmed HBSS, and then depolarized in hyperkalemic HBSS containing $15 \mu \mathrm{M}$ FM4-64 (Invitrogen) for $90 \mathrm{~s}$ followed by regular HBSS containing $15 \mu \mathrm{M}$ FM4-64 for $2 \mathrm{~min}$. Cells were then washed several times with regular HBSS containing $1 \mathrm{mg} / \mathrm{ml}$ Advasep (Cydex) and allowed to rest $5 \mathrm{~min}$ in HBSS-Advasep, during which the first series of images were acquired. The HBSS-Advasep was then removed and replaced with hyperkalemic HBSS for $90 \mathrm{~s}$ to facilitate destaining of hippocampal terminals. Cells were washed with plain HBSS several times and imaged once again.

For experiments using EGFP-SV2-infected neurons, cells were first incubated with beads for defined time points ( 1 and $24 \mathrm{~h}$ shown here) in glial-conditioned medium at $37^{\circ} \mathrm{C}, 5 \% \mathrm{CO}_{2}$, then mounted onto the microscope stage in Air medium for selection of EGFP-SV2-positive bead sites. We used a motorized stage to sample from several different sites on a single coverslip.

EGFP-SV2-positive presynaptic boutons were labeled with the FM4-64 dye by perfusion first with Tyrode's saline solution (Leal-Ortiz et al., 2008 ), followed by incubation in high- $\mathrm{K}^{+}$Tyrode's solution (Tyrode's + $90 \mathrm{~mm} \mathrm{KCl}, 31.5 \mathrm{~mm} \mathrm{NaCl}$ ) containing $\sim 1 \mu \mathrm{g} / \mathrm{ml} \mathrm{FM}$ dye for $60 \mathrm{~s}$, followed by Tyrode's + FM dye for $30 \mathrm{~s}$. Neurons were then washed for 5 min in normal Tyrode's before imaging. Destaining was performed by perfusion with high- $\mathrm{K}^{+}$Tyrode's for $60 \mathrm{~s}$ followed by a $2 \mathrm{~min}$ washout in normal Tyrode's before acquisition of the destained images.

Image quantification and analysis. Quantification of immunocytochemistry and colocalization was performed using NIH ImageJ software. All quantifications were calculated for at least 30 beads per condition per experiment and averaged across a minimum of three separate experiments per condition. All brackets beneath histograms show the total number of beads analyzed. Values in histograms are always expressed as mean \pm SEM.

To quantify the recruitment of synaptic proteins under the beads, the brightfield (DIC) image was used to locate and highlight each bead contacting a neurite, and for each bead, an adjacent equal-sized area was highlighted to act as a control. Great care was taken to ensure that the adjacent site contained a similar density of neurites with respect to the corresponding bead site to be analyzed. Beads contacting cell bodies were not included in the analysis. Fluorescence was quantified by measuring either (1) the fluorescence intensity at each bead and corresponding adjacent contact or (2) the proportion of labeled pixels at each bead and adjacent contact following careful thresholding. Values were expressed as bead/adjacent fluorescence intensity ratios or fluorescence ratios, respectively. For the colocalization analysis (Fig. $1 G$ ), we used the Intensity Correlation Analysis (ICA) plugin within the NIH ImageJ program (Li et al., 2004).

Quantification of the lentiviral-infected cultures imaged by live time lapse was performed using MetaMorph software (Molecular Devices). 
A
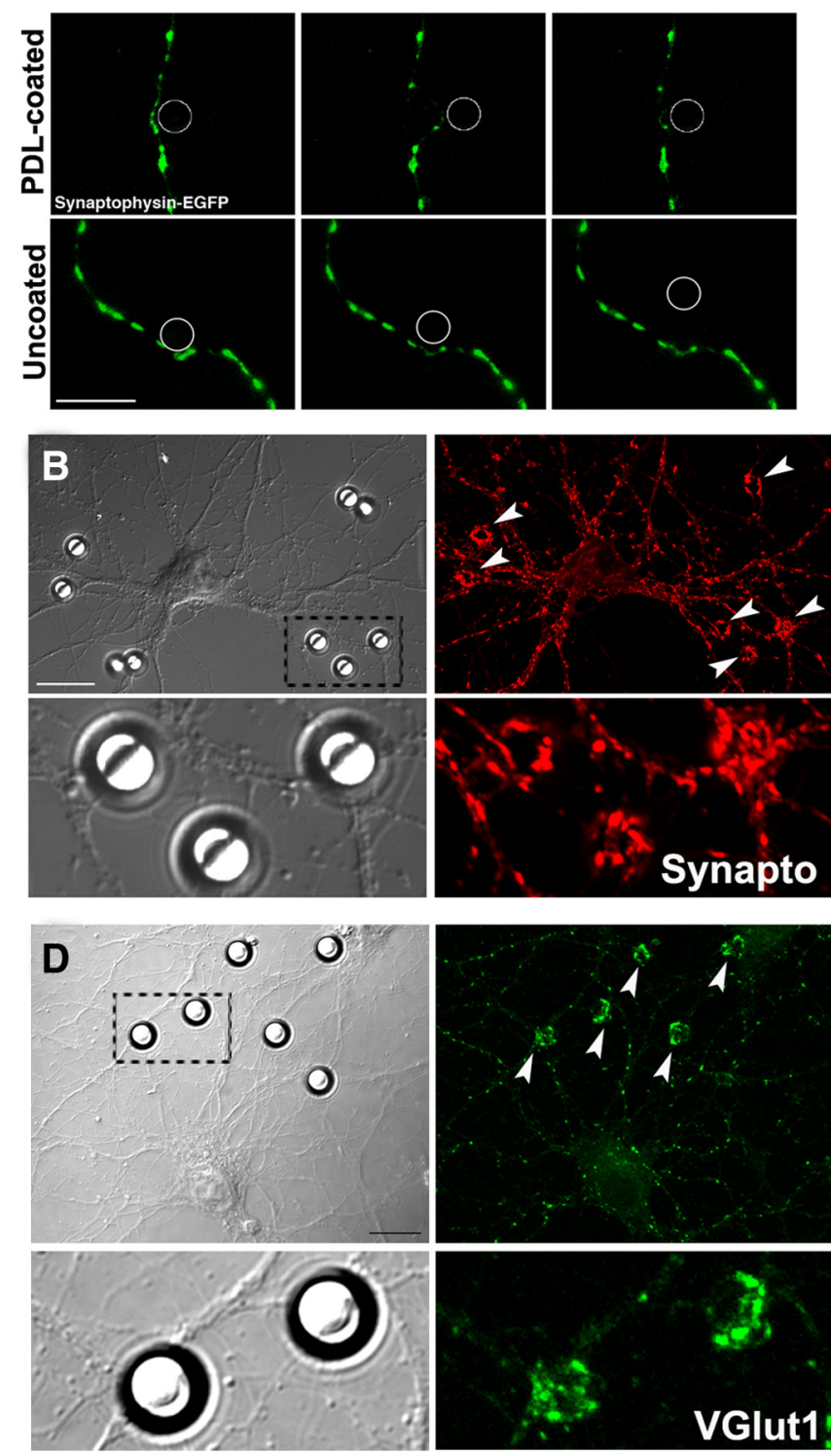

E

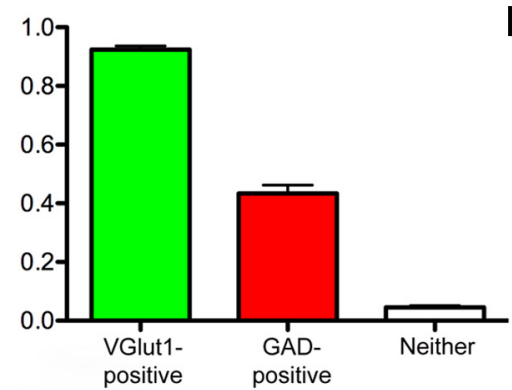

$\mathbf{F}$
C
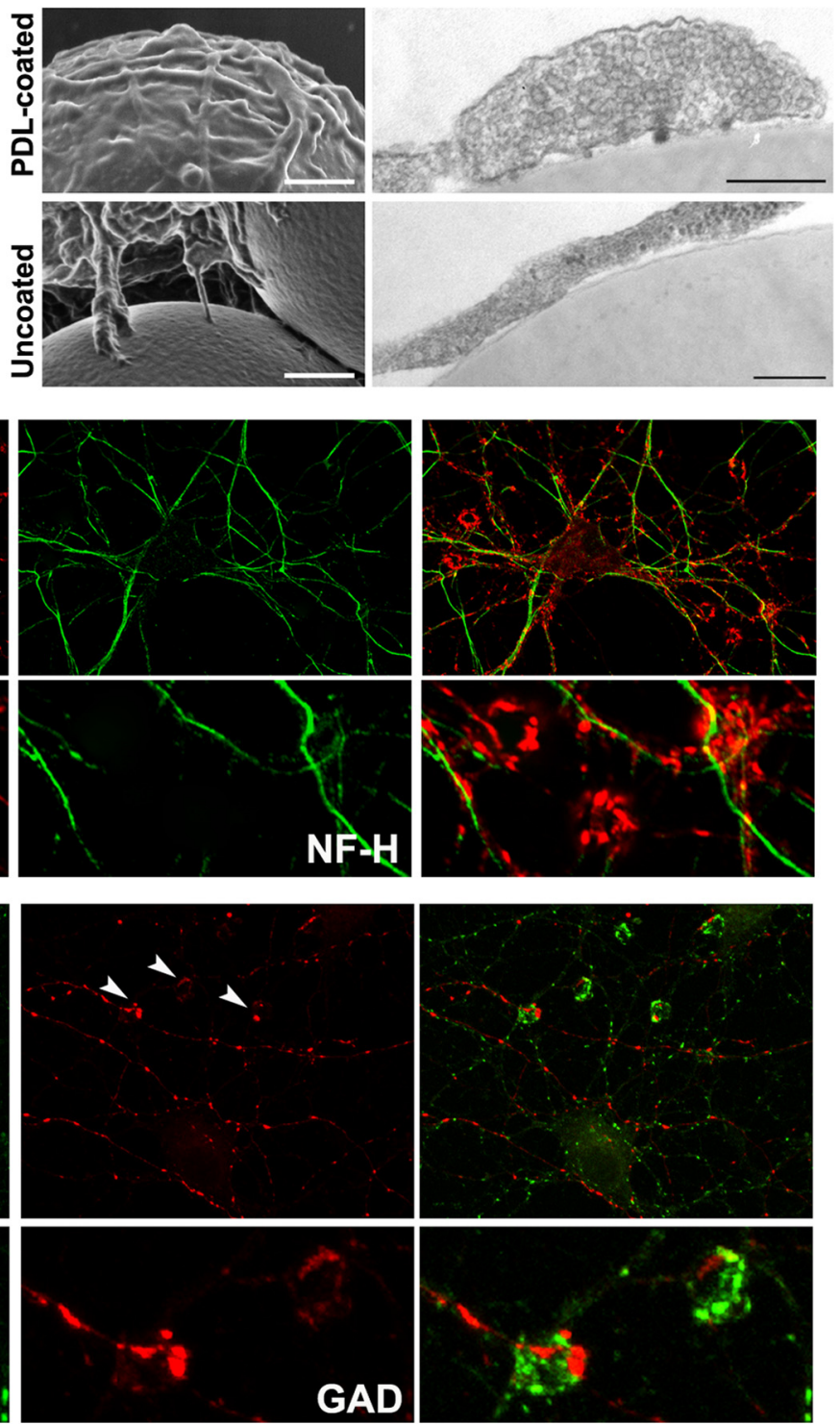

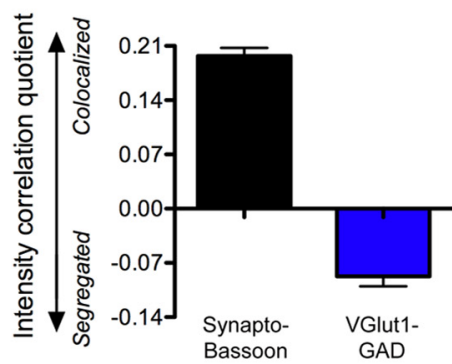

Figure 1. PDL-coated beads induce the formation of adherent synaptic vesicle complexes on axons. A, Bioscope-AFM images of axon-bead contacts (white circles). Top, PDL-coated bead in contact with a synaptophysin-EGFP-transfected axon for $2 \mathrm{~h}$. Note how the axon follows the movement of the PDL bead via the cantilever to which the bead is attached, indicating a significant adhesion. Bottom, An uncoated bead manipulated in the same way results in the immediate detachment of bead from the axon, revealing that no significant adhesion had taken place. Scale bar, $10 \mu \mathrm{m}$. B, DIV15 rat hippocampal neurons incubated with PDL-coated beads for $24 \mathrm{~h}$ (DIC, far left) labeled with antibodies to heavy chain neurofilament (green) and synaptophysin (red). Bottom, Zoom of the box shown in the larger DIC image. Scale bar, $20 \mu \mathrm{m}$. C, Scanning EM (left) and TEM (right) images of neurons cocultured for $24 \mathrm{~h}$ with PDL-coated (top) or uncoated (bottom) beads. By scanning EM, we observed that neurites extend dense and numerous processes onto PDL-coated beads (top left), but fail to extend processes onto uncoated beads (bottom left). By TEM, we find that these dense processes contain bouton-like swellings and accumulate synaptic vesicles when contacting PDL beads (top right), whereas neurites contacting uncoated beads do not form these varicosities (bottom right). Scale bars: $1 \mu \mathrm{m}$ (scanning EM), $250 \mathrm{~nm}$ (TEM). D. DIV15 hippocampal neurons incubated with PDL-coated beads for $24 \mathrm{~h}$ labeled with antibodies to VGlut1 (green) and GAD (red). The dashed box in the full DIC image (top left) corresponds to the location of the close-up panel (bottom). Scale bar, $20 \mu \mathrm{m}$. $\boldsymbol{E}$, Proportion of bead contacts displaying enhancement (fluorescence intensity ratio $>2$ ) of GGlut1 or GAD.F, Colocalization ofVGlut1 and GAD puncta at bead sites. Compared with the distributions of synaptophysin and bassoon (black bar), which are highly covariant, we observe that the VGlut1 and GAD staining distributions are segregated from one another even when on the same bead (blue bar), as would be expected if these puncta are derived from different axons. 
To measure changes in fluorescence intensity, we performed line scans of axonal/dendritic profiles with or without beads and measured their fluorescence intensities for each time point acquired. All individual values were thereafter normalized to the average baseline fluorescence intensity values for each site (before the addition of the beads), plotted, and statistically analyzed.

All images were processed and prepared for print using Photoshop (Adobe).

Statistics. All statistics were performed and data graphed using GraphPad Prism software. For comparisons of fluorescence changes between two groups, we assessed significance using Student's $t$ test. For comparisons between multiple groups we used one-way ANOVA followed by Bonferroni's post hoc test. To assess changes in fluorescence intensity with time (live imaging data, see Fig. 5), we performed two-way ANOVA with time as the repeated measure. All data shown are mean \pm SEM. In figures, statistical significance is indicated by \#/n.s. for $p>0.05,{ }^{*}$ for $0.05>p>$ $0.01,{ }^{* *}$ for $0.01>p>0.001$, and ${ }^{* * *}$ for $p<0.001$.

\section{Results}

PDL-coated beads induce membrane adhesion followed by the subplasmalemmal clustering of synaptic vesicle complexes under the adhesion site within axons

Previous studies (Burry, 1980, 1982; Peng et al., 1987) revealed that cultures of cerebellar granule cells were capable of forming contact-induced clusters of vesicles within $24 \mathrm{~h}$ on PLL-coated beads. To further investigate this phenomenon, low-density hippocampal cultures were grown (to DIV7, DIV15, or DIV21) and thereafter incubated with $7 \mu \mathrm{m}$ polystyrene beads coated with PDL $(50 \mu \mathrm{g} / \mathrm{ml})$ (supplemental Fig. S1, available at www. jneurosci.org as supplemental material). PDL is an isomer of PLL, which, like PLL, is typically used to promote in vitro adhesion of cells to a substrate. Incubation of these beads with cells for as long as $72 \mathrm{~h}$ appeared to have no adverse affect on neuron health or on their ability to arborize their axons or dendrites as assessed by either differential interference contrast (DIC) microscopy (Fig. $1 B$; supplemental Fig. S1, available at www.jneurosci. org as supplemental material) or immunofluorescence with antibodies to neurofilaments (NF-H, see Fig. $1 B$ ).

When PDL-coated beads were added to cultured hippocampal neurons, we were first struck by how the beads adhered to cell surface membrane domains within minutes, and were resistant to mechanical dislodgment throughout the incubation period. In contrast, uncoated beads mostly remained free in the culture medium even after several hours, and were readily washed away after fixation. To assess the "attachment phase" more directly, we combined live imaging with AFM. In these experiments, neurons expressing synaptophysin-EGFP [to mark neuronal processes] were exposed to PDL-coated or uncoated beads immobilized on a cantilever attached to a Bioscope-AFM. When an immobilized PDL-coated bead was gently placed against a synaptophysinEGFP-expressing axonal membrane, the bead became resistant to detachment within just a few minutes of contact (data not shown). After a $2 \mathrm{~h}$ incubation period, moving the cantilever attached to a PDL-coated bead resulted in a corresponding displacement of the axon several micrometers from the site of the culture dish onto which the axon was attached, thus revealing a strong, grossly observable adhesion event (Fig. 1A, top; supplemental Movie S1, available at www.jneurosci.org as supplemental material). In contrast, moving the cantilever bound to an uncoated bead resulted in its immediate detachment from the axon even after $30 \mathrm{~min}$ of contact, revealing no significant adhesive interaction between uncoated beads and axons (Fig. 1A, bottom; supplemental Movie S2, available at www.jneurosci.org as supplemental material).
The ability of the beads to induce clustering of synaptic vesicles (SVs) was assessed by determining the localization pattern of the synaptic vesicle protein synaptophysin. Following a $24 \mathrm{~h}$ bead incubation, we observed a marked enhancement of synaptophysin immunolabeling at sites of contact between neurites and PDL-coated beads in the vast majority of neurons visualized at all three stages of maturity tested (Fig. $1 B$; supplemental Fig. S1, available at www.jneurosci.org as supplemental material). Prominent synaptophysin-positive puncta were also found in association with PDL beads following a $72 \mathrm{~h}$ incubation period, indicating that these bead-induced SV clusters are quite stable (supplemental Fig. S1, available at www.jneurosci.org as supplemental material).

The morphological features of these presynaptic-bead complexes (following $24 \mathrm{~h}$ of incubation) were next assessed by electron microscopy. By scanning EM, we observed dense and profuse neurite extensions onto PDL-coated beads (Fig. 1C, top left) but not on uncoated beads (Fig. $1 C$, bottom left). TEM revealed that processes extending onto PDL-coated beads contained bouton-like swellings filled with $50 \mathrm{~nm}$ vesicles (Fig. 1C, top right), while vesicle clusters were not observed in neurites contacting uncoated beads (Fig. $1 C$, bottom right).

We next evaluated whether SV clusters were derived from the axons of inhibitory and/or excitatory neurons. To this end, cultures were incubated with beads for $24 \mathrm{~h}$ then immunostained with antibodies against the vesicular glutamate transporter 1 (VGlut1) or glutamic acid decarboxylase (GAD). These experiments revealed that a majority of the SV clusters were VGlut1-positive ( $~ 90 \%)$, while a smaller proportion were immunopositive for GAD $(\sim 40 \%)$ (Fig. $1 D, E)$. Quantitative analysis revealed that in each case, GAD-positive puncta were not VGlut1 immunoreactive, and vice versa, indicating that each arises independently from either GAD- or VGlut1-positive axons, respectively (Fig. 1D,F). Although there were fewer GAD-positive boutons, where present, they displayed enhanced clustering to the same degree as VGlut1 (fluorescence intensity ratios: VGlut1, $7.407 \pm 1.360$ vs $\mathrm{GAD}, 8.766 \pm 0.337 ; p=0.39)$. This result suggests that PDLcoated beads robustly induce the clustering of SVs from both excitatory and inhibitory neurons.

\section{PDL-coated beads induce the formation of functional presynaptic boutons}

Although the SV clustering phenomenon had been described previously (Burry, 1980, 1982; Peng et al., 1987), it was unclear whether or not these bouton-like structures contained active zones indicative of functional bona fide presynaptic endings. This was first explored by immunostaining cultures of hippocampal neurons, grown for $15 \mathrm{DIV}$, and incubated with beads for $24 \mathrm{~h}$, with antibodies against a variety of SV and active zone markers (Fig. 2A, Table 1). In addition to synaptophysin (Fig. 2Ai), we also observed a robust enhancement of immunoreactivity for the active zone proteins bassoon (Fig. 2Aii), rab3ainteracting molecule (RIM) (Fig. 2Aiii), the N-type calcium channel CaV2.2 (Fig. 2Aiv), N-cadherin (Fig. 2Av), and F-Actin (Alexa-phalloidin) (Fig. 2Avi) on beads coated with PDL but not uncoated beads. There was also a small but significant enhancement of tubulin at PDL-coated but not at uncoated bead contacts, suggesting that PDL-coated beads may induce changes in microtubular organization (Fig. 2Avii, Table 1) (cf. Dillon and Goda, 2005). Alternatively, the accumulation of tubulin at PDL bead sites may be accounted for by the small increase in axonal contact area at PDL bead but not uncoated bead contacts, as determined from the expression of soluble EGFP along trans- 
A
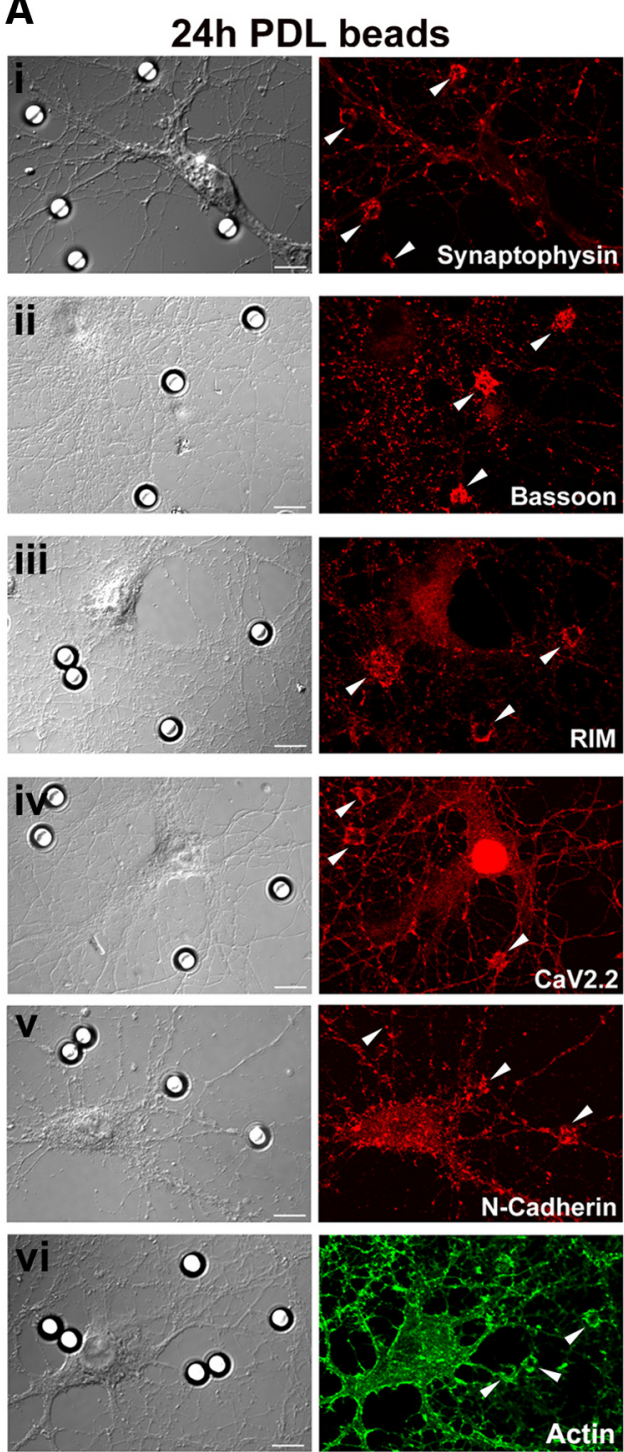

o
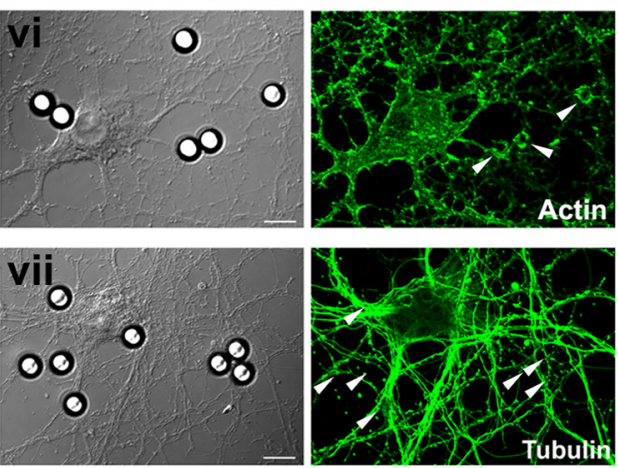

B
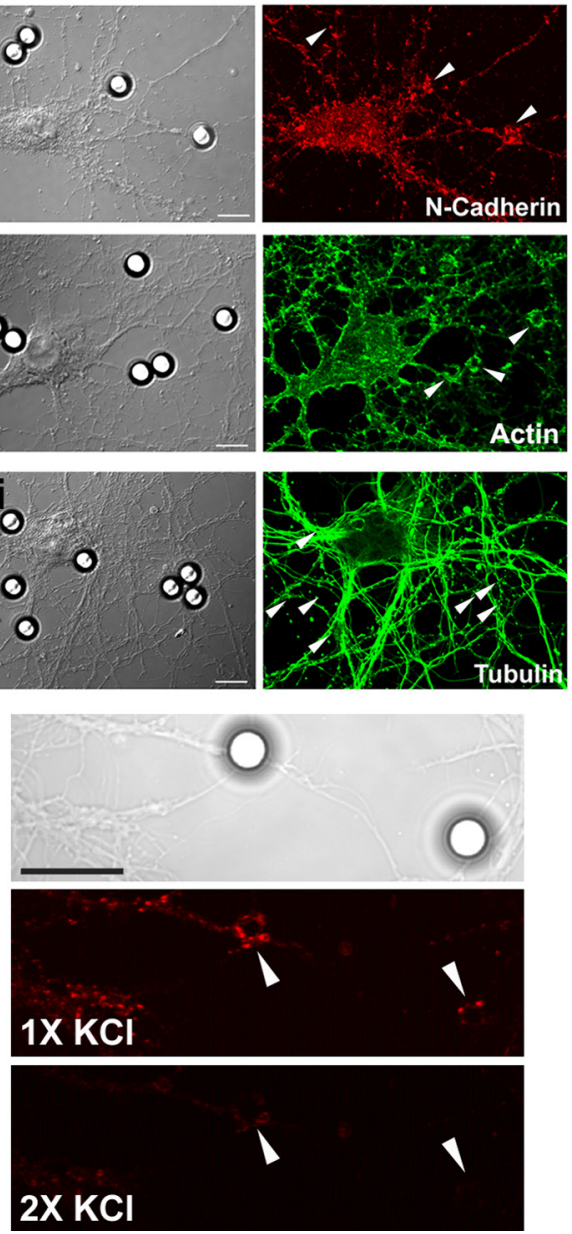

24h Uncoated beads
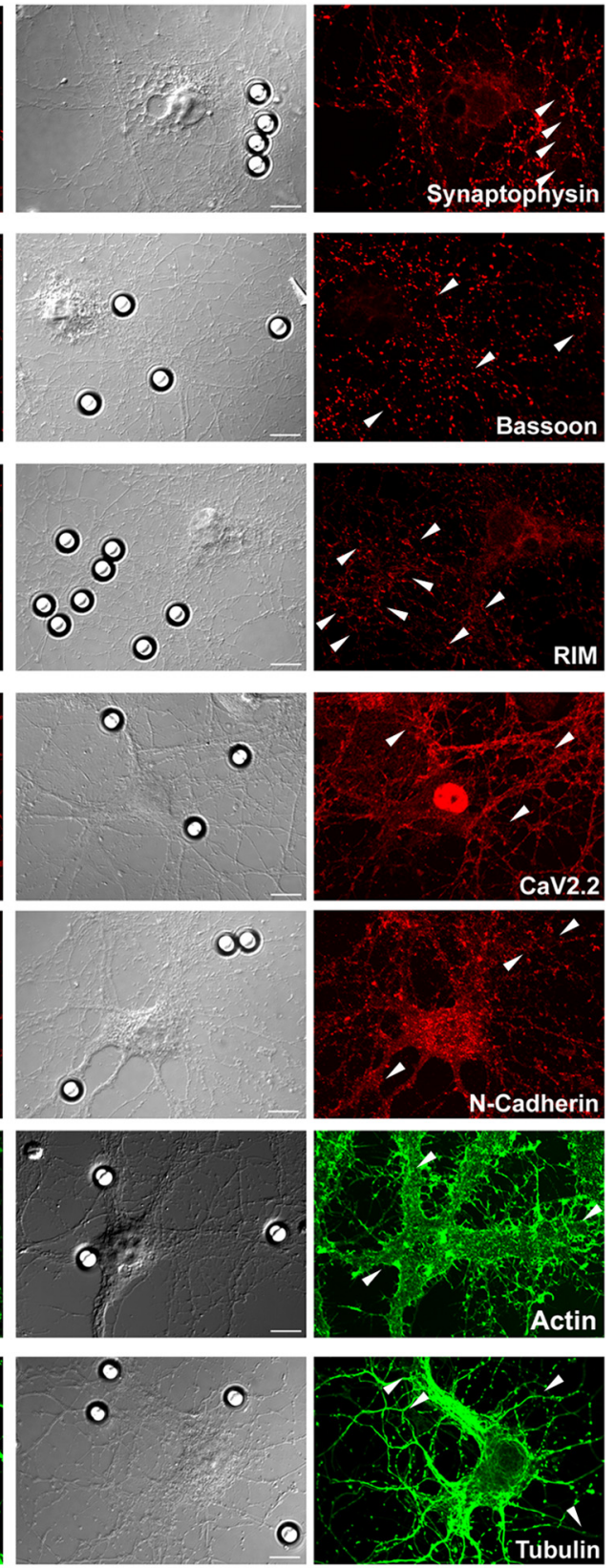

C

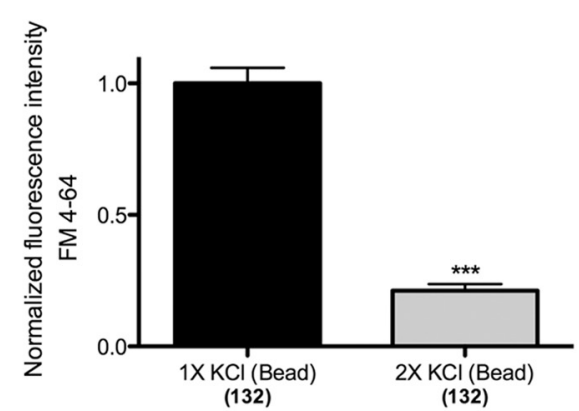

Figure 2. PDL-coated beads induce the formation of functional presynaptic boutons. $\boldsymbol{A}$, DIV15 neurons incubated for $24 \mathrm{~h}$ with either PDL-coated (left image) or uncoated (right image) beads were fixed and immunostained for a variety of presynaptic and cytoskeletal markers ( $\boldsymbol{i}$-vii). For each fluorescence image, the corresponding DIC image is represented on the left to note the location of the bead. Scale bars, $10 \mu \mathrm{m}$. Arrowheads denote the bead sites. B, Representative image of (DIV15) neurons cocultured with beads for $24 \mathrm{~h}$ (phase-contrast image, top) (Figure legend continues.) 
fected axons [ratios: $2.38 \pm 0.48(\mathrm{PDL})^{\star *}$ vs $1.06 \pm 0.08$ (uncoated)] (supplemental Fig. S2, available at www.jneurosci.org as supplemental material). Although significant, this increase cannot account for the $>10$-fold increases in the clustering of synaptophysin, RIM, and bassoon (Table 1). Together, these data reveal that these axonal swellings contain more than just clusters of SVs, and in fact may be functional presynaptic boutons.

To assess whether these presynaptic-like endings could recycle SVs in an activity-dependent manner, we depolarized neuronbead cultures (DIV15 + 24 h PDL-coated beads) with $\mathrm{KCl}(45$ $\mathrm{mM}$ ) in the presence of the styryl dye FM4-64 (Ryan et al., 1993; Ryan and Smith, 1995). This treatment led to the appearance of fluorescent puncta along dendritic profiles, confirming the presence of functional presynaptic boutons at native axodendritic contacts. We also observed the appearance of fluorescent puncta along the perimeter of PDL-coated beads in contact with axons (Fig. 2B). Exposure to a second depolarizing concentration of $\mathrm{KCl}$, to cause exocytosis of dye-filled SVs, resulted in a significant decrease in the intensity of fluorescent puncta along dendrites as well as neurite-PDL bead contact sites (Fig. 2B,C). Together, these data demonstrate that PDL-coated beads induce the formation of functional presynaptic boutons, closely resembling the presynaptic compartments assembled as a result of induction by natural substrates.

\section{PDL induces the formation of presynaptic endings through a mechanism involving HSPGs}

PDL is typically used to coat substrates to promote cell adhesion in vitro (Yavin and Yavin, 1974). In previous studies, it had been reported that isolated axons grown on a PDL-coated substrate form presynaptic-like structures similar in composition to the bead-presynaptic complexes reported here, but these structures were highly mobile and not thought to be triggered by the substrate itself (Krueger et al., 2003). Although our observations suggest that PDL-coated beads induce highly stable presynaptic complexes regardless of age or culture density (supplemental Fig. S1, available at www.jneurosci.org as supplemental material), both the dish and the beads were coated with PDL in our experiments; therefore, it remained possible that these two types of structures were somehow related.

To address this question, we devised the following experiment (Fig. 3A). Dissociated hippocampal cells were plated on substrates already containing PDL-coated beads and allowed to differentiate in their presence for several days (DIV0 to at least DIV7). Then, we added a second population of beads, this time coated with a FITC-conjugated version of polylysine (PLL-FITC) to allow us to distinguish between the two bead populations. In control experiments, we found PLL-FITC to be as effective as PDL at inducing presynaptic complexes when either type of bead is added to cultures after DIV7 (data not shown). However in this experimental setup, where beads were added to cultures both before and after the developmental stage in vitro in which they become competent to form synapses, only the PLL-FITC beads induced the formation of presynaptic clusters, while the PDL beads added at DIV0 did not (Fig. $3 B$ ). This result suggests that

\footnotetext{
$\leftarrow$

(Figure legend continued.) before depolarization-induced loading ( $1 \times \mathrm{KCl}$, middle) and unloading $(2 \times \mathrm{KCl}$, bottom) of the synaptic dye FM4-64. Arrowheads denote the bead sites. Scale bar, $20 \mu \mathrm{m}$. C, Histogram showing measurements of fluorescence intensity following dye loading $(1 \times \mathrm{KCl})$ and unloading $(2 \times \mathrm{KCl})$ at bead contacts $(20-30$ beads per experiment, $n=5$ experiments, $\left.{ }^{* * *} p<0.001\right)$.
}

Table 1. Quantification and analysis of immunofluorescence using presynaptic and cytoskeletal markers

\begin{tabular}{|c|c|c|c|c|}
\hline & \multicolumn{2}{|c|}{$\begin{array}{l}\text { Fluorescence ratios } \\
24 \mathrm{~h} \text { PDL beads }\end{array}$} & \multicolumn{2}{|c|}{$24 \mathrm{~h}$ Uncoated beads } \\
\hline & Mean \pm SEM & $\begin{array}{l}\text { Total \# beads } \\
(n=3 \text { expts.) }\end{array}$ & Mean \pm SEM & $\begin{array}{l}\text { Total \# beads } \\
(n=3 \text { expts.) }\end{array}$ \\
\hline Synaptophysin & $12.9 \pm 1.3^{* * *}$ & 423 & $1.2 \pm 0.1$ & 287 \\
\hline Bassoon & $24.4 \pm 2.9^{* * *}$ & 200 & $1.6 \pm 0.1$ & 132 \\
\hline RIM & $21.7 \pm 3.9^{* * *}$ & 223 & $1.9 \pm 0.1$ & 155 \\
\hline CaV2.2 & $7.4 \pm 1.1^{* * *}$ & 277 & $1.6 \pm 0.2$ & 138 \\
\hline $\mathrm{N}$-cadherin & $10.0 \pm 1.8^{* *}$ & 299 & $1.5 \pm 0.1$ & 177 \\
\hline Actin & $7.4 \pm 1.4^{*}$ & 319 & $1.2 \pm 0.1$ & 99 \\
\hline Tubulin & $1.6 \pm 0.1^{*}$ & 214 & $1.2 \pm 0.1$ & 148 \\
\hline
\end{tabular}

Values represent the average fluorescence ratios at PDL-coated or uncoated bead sites for the presynaptic and cytoskeletal markers listed. The corresponding representative fluorescence images are shown in Figure $2 \mathrm{Ai}-\mathrm{vii}$. ${ }^{* * *} p<0.001,{ }^{* *} p<0.01,{ }^{*} p<0.05$, PDL versus uncoated beads, Student's $t$ test.

neurons must reach a stage of development at which they are competent to form synapses in order for polylysine to behave as a synaptogenic substrate, and before this, that PDL behaves primarily as a promoter of axon outgrowth and cell adhesion. Furthermore, we conclude that these presynaptic complexes induced by PDL beads are unique, both functionally and morphologically, compared with the presynaptic-like structures reported previously (Krueger et al., 2003).

PDL is a highly cationic artificial polymer. How might it induce the formation of functional presynaptic compartments? We observed that the accumulation of synaptophysin increases at bead contacts with increasing concentrations of PDL on the bead surface (Fig. 3C), clearly indicating that this protein, and not the bead itself, is responsible for the observed effects. However, this clustering effect is markedly reduced in cultures incubated with beads coated with cationic lipids (Fig. 3D). To confirm that the concentration of positive charge was the same, we performed a $\zeta$-potential analysis to analyze overall surface charge and found no difference between PDL- and lipid-coated beads $(50 \mu \mathrm{g} / \mathrm{ml}$ PDL beads, $46 \pm 3 \mathrm{mV}$ vs lipid beads, $45 \pm 3 \mathrm{mV}$; data not shown). These results indicate that positive charge alone is insufficient to drive presynaptic assembly, suggesting that the configuration of charge on the PDL surface might be instead the defining characteristic that drives its inductive properties.

We then speculated that perhaps a direct interaction between some extracellular or transmembrane component and the PDL is responsible for presynaptic compartment induction. Although charge alone is not responsible for its effects, PDL is nevertheless poised to interact with substances that are negatively charged in an electrostatic manner. HSPGs are a large and heterogeneous family of extracellular and transmembrane molecules which in part comprise the architecture of the extracellular matrix, and a growing body of literature points to a role for these molecules in synapse formation as well as other critical stages of neuronal and synaptic development, including axon guidance and synaptic plasticity (Rauvala and Peng, 1997; Lauri et al., 1999; Bandtlow and Zimmermann, 2000; Dityatev et al., 2004; Dityatev and Schachner, 2006; Johnson et al., 2006; Van Vactor et al., 2006; Matsumoto et al., 2007). HSPGs are composed of a proteoglycan core posttranslationally modified to provide anionic sites for a number of extracellular binding partners (Gallagher et al., 1986). Given that these molecules are heavily negatively charged, they have been predicted to be good substrates for interaction with molecules rich in positively charged residues such as lysines and arginines (Cardin and Weintraub, 1989). Furthermore, it has been shown in heterologous cell lines that short cationic 
A

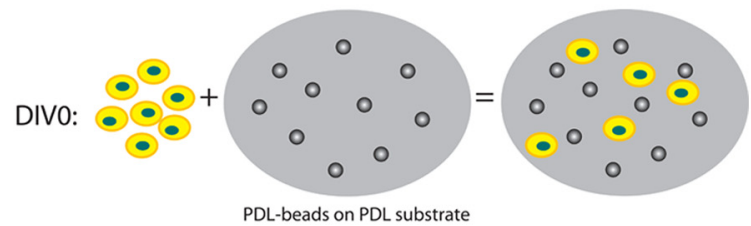

PDL-beads on PDL substrate
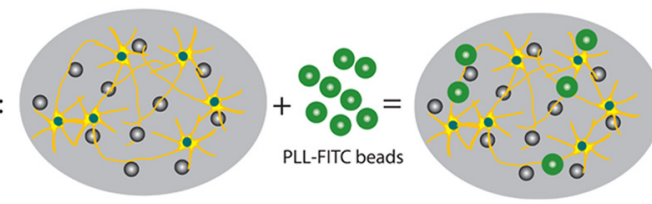

B
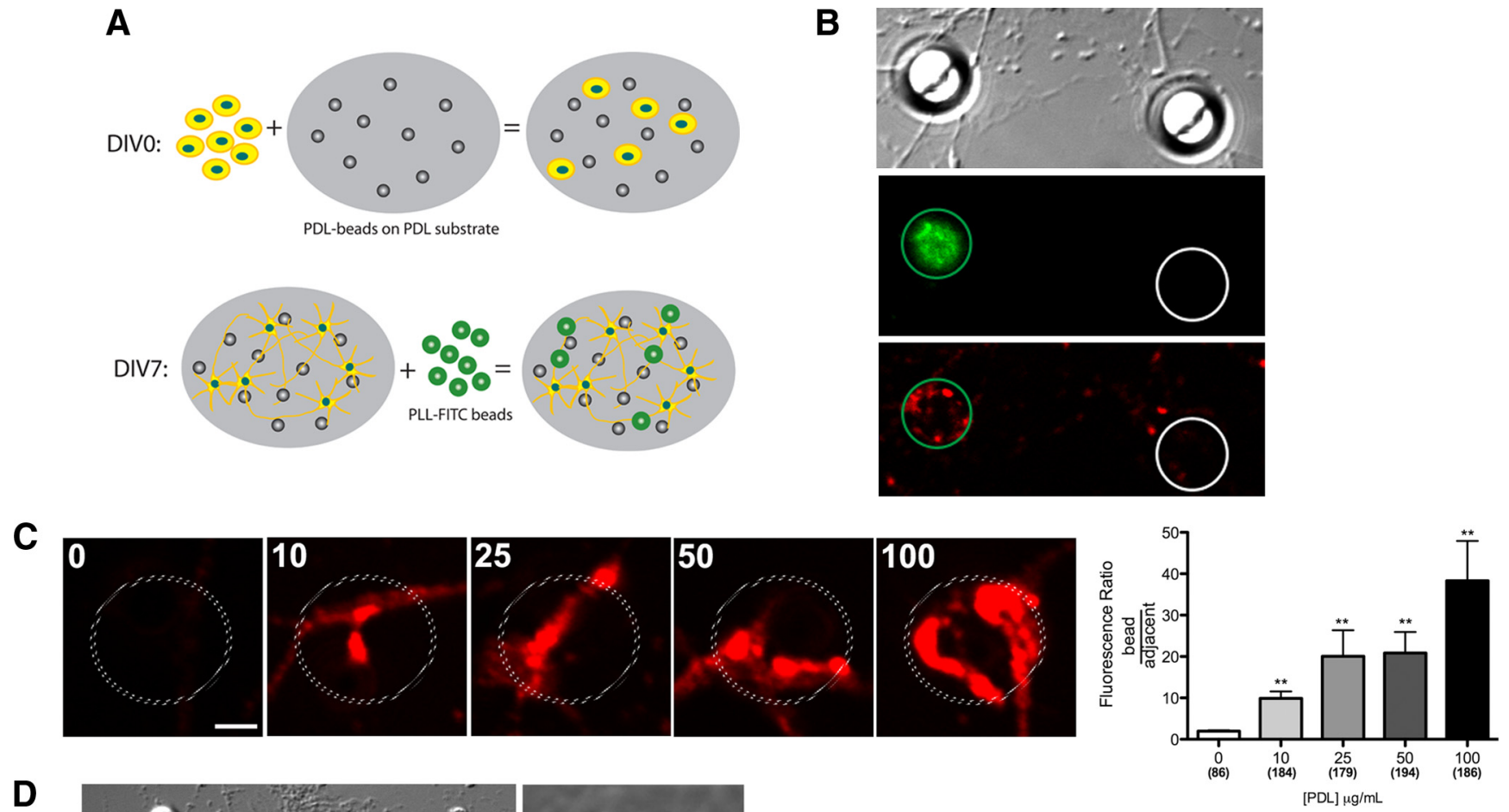

D
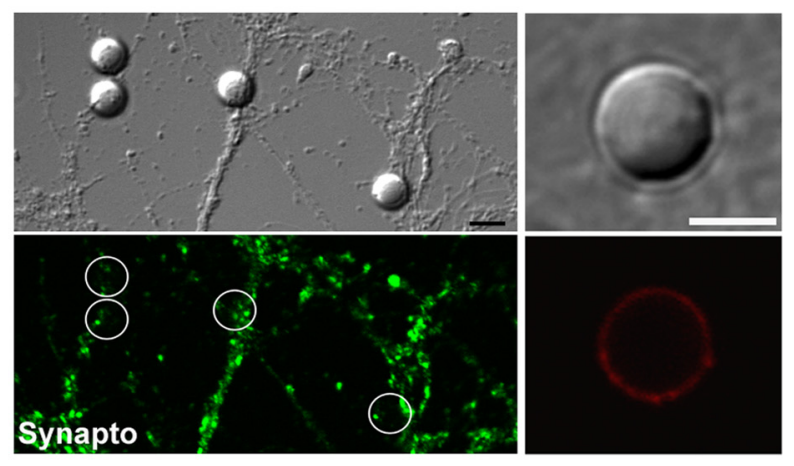

E
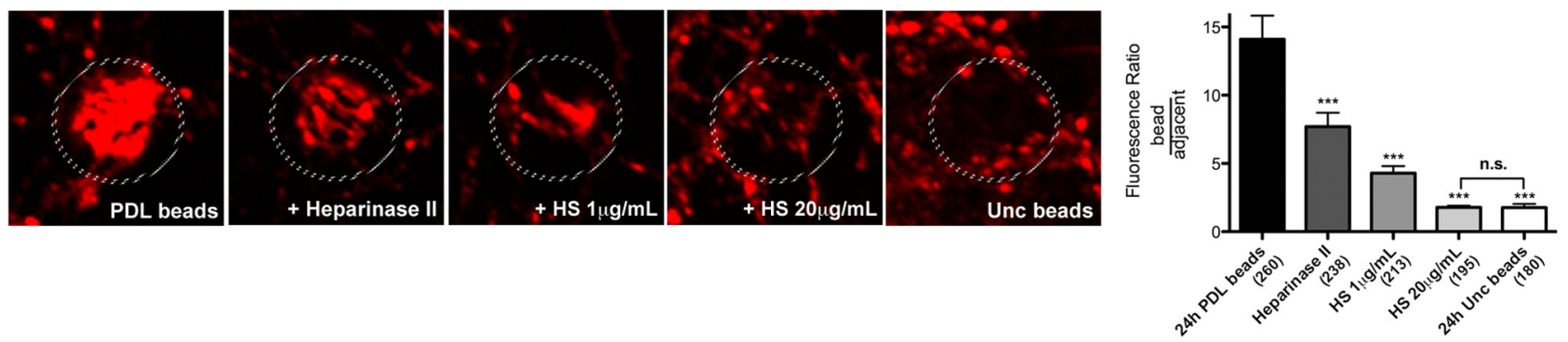

Figure 3. PDL bead-induced presynaptic boutons are different from isolated axonal clusters, and form in a dose-dependent manner by a mechanism involving HSPGs. $A, B$, Addition of beads at different developmental stages distinguishes inductive capacity of PDL on the substrate versus the bead. $A$, Experimental design. At the time of plating, dissociated hippocampal cells were added to a PDL-coated substrate containing PDL-coated beads. After 7 DIV, PLL-FITC-coated beads were added and left for $24 \mathrm{~h}$, fixed, and imaged as usual. B, DIC and fluorescence images of synaptophysin (red) and PLL-FITC beads (green). Only the PLL-FITC-coated bead (green circle) induces synaptophysin clusters, while the PDL-coated bead added at the time of plating (white circle) does not. C, Representative images of DIV7 axons contacting beads coated with increasing concentrations of PDL for $24 \mathrm{~h}$. Histogram, Measurements of fluorescence at neurite- bead contacts with increasing PDL concentrations $\left({ }^{* *} p<0.01\right)$. Scale bar, $2 \mu \mathrm{m}$. Dashed circles indicate the location of the beads. $\boldsymbol{D}$, Beads coated with a synthetic lipid bilayer containing DOTAP, a cationic lipid, were incubated with neuronal cultures (DIV12-DIV14) for $24 \mathrm{~h}$. Left, Representative images of cultures incubated with DOTAP beads (DIC, top left) and immunolabeled for synaptophysin (green, bottom left). Scale bar, $10 \mu \mathrm{m}$. Far right, DOTAP beads with rhodamine (red, bottom right) incorporated into the lipid bilayer confirm that the bilayer remains intact when incubated with neurons for $24 \mathrm{~h}$. Scale bar, $5 \mu \mathrm{m}$. $\boldsymbol{E}$, Representative images of neurons (DIV15) incubated for $24 \mathrm{~h}$ with PDL or uncoated beads with or without heparinase or heparan sulfate. Histograms, Fluorescence ratio measurements in response to the different treatments $\left({ }^{* *} p<0.001 ; \mathrm{n}\right.$.s. between $20 \mu \mathrm{g} / \mathrm{ml}$ heparan sulfate and uncoated beads). Scale and circles are the same as in $\boldsymbol{C}$.

peptides and/or motifs bind to and can drive intracellular signaling through interaction with cell-surface HSPGs (Mislick and Baldeschwieler, 1996; Chang et al., 1997; Nakase et al., 2007). We therefore hypothesized that HSPGs, which are known to be expressed in hippocampal cultures in both transmembrane and secreted form (Dow et al., 1988; Sugiura and Dow, 1994; Hsueh and Sheng, 1999), were potential endogenous targets for interaction with PDL-coated beads.

We next evaluated whether HSPGs direct the formation of presynaptic endings onto PDL-coated beads. In the first of these experiments, neurons grown for 15 DIV were incubated with PDL beads for $24 \mathrm{~h}$ in the presence of heparinase II, an enzyme 
A

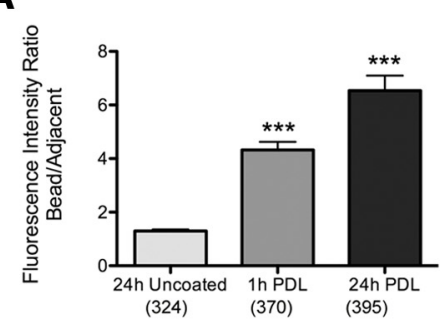

C
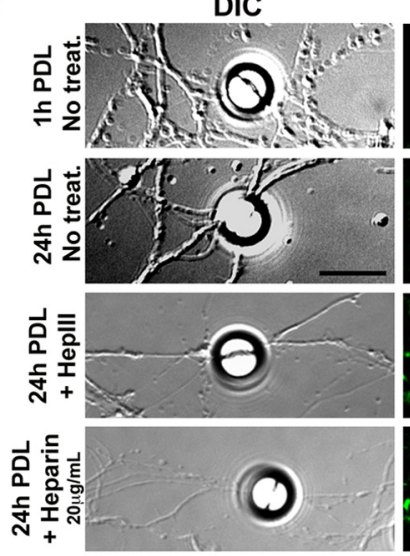

D

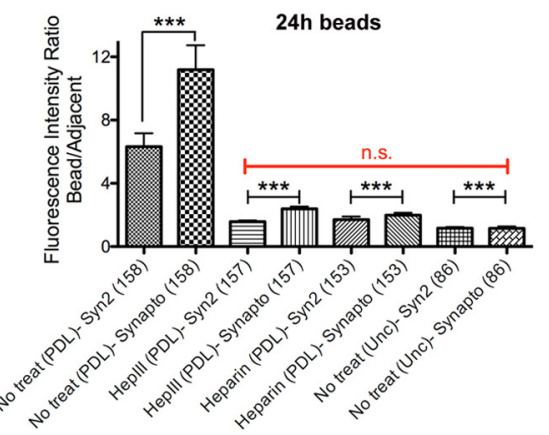

B

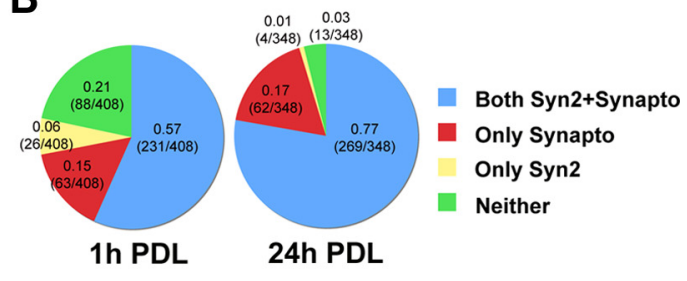

PDL-coated beads depend on HSPGs to facilitate the contact-mediated de novo assembly of presynaptic boutons.

Which HSPGs may be involved in this phenomenon? To address this question, we first asked whether HSPGs themselves could cluster directly onto the beads. Using a pan-heparan sulfate antibody that recognizes an epitope present in a variety of HSPGs (10E4 epitope), we found significantly enhanced HS immunofluorescence at PDL bead sites compared with uncoated bead sites (supplemental Fig. S4A, $C$, available at www.jneurosci.org as supplemental material). This is consistent with the increase in synaptophysin found exclusively at PDL bead sites (supplemental Fig. $\mathrm{S} 4 B, C$, available at www.jneurosci. org as supplemental material). Next, we wished to assess the role of specific HSPGs. We focused our attention on syndecan-2 (syn2), a cell-surface HSPG shown previously to cluster at synapses both presynaptically and postsynaptically (Hsueh et al., 1998; Ethell and Yamaguchi, 1999; Hsueh and Sheng, 1999; Ethell et al., 2001). We found a significant enhancement of syn 2 at PDL bead sites within $1 \mathrm{~h}$ as well as after $24 \mathrm{~h}$ of contact (Fig. $4 A, C$ ). When we dually immunostained cultures for syn2 along with synaptophysin, we found that the vast majority of bead sites displayed enhanced coclustering of both proteins (Fig. $4 B, C$ ). It should be noted that after $1 \mathrm{~h}$, a sizeable proportion of bead sites expressed neither syn2 nor synaptophysin, while a second smaller proportion expressed enhancement of synaptophysin only. This latter population persisted at the $24 \mathrm{~h}$ time point, suggesting that syn2 does not become clustered in a small proportion of PDL bead sites (Fig. $4 B$ ). However, after $24 \mathrm{~h}$, the proportion of sites that displayed no enhanced clustering was markedly reduced, appearing to be replaced by a larger proportion that was dually enhanced for both proteins. This analysis reveals a correlated accumulation of the cell surface HSPG syndecan-2 and synaptophysin at a majority of PDL bead

that degrades HSPGs. Here, we found a dramatic decrease in the intensity of synaptophysin puncta associated with PDL beads (Fig. 3E). Similarly, we observed a dose-dependent decrease in the number and intensity of synaptophysin puncta when PDL beads were added in the presence of $1 \mu \mathrm{g} / \mathrm{ml}$ or $20 \mu \mathrm{g} / \mathrm{ml}$ heparan sulfate, a condition predicted to compete with the binding of endogenous HSPGs to PDL (Fig. 3E, histogram). These treatments did not affect the binding of PDL beads to the axonal surface. Furthermore, we did not find any overall changes in presynaptic puncta size, number, or intensity at nonbead sites, suggesting that these treatments did not have any effect on established boutons (supplemental Fig. S3, available at www.jneurosci. org as supplemental material). Together, these data indicate that sites, suggesting that perhaps syn 2 is involved in the triggering of PDL bead-induced presynapse formation.

To better address this question, we incubated cultures with PDL beads in the presence of heparinase or $20 \mu \mathrm{g} / \mathrm{ml}$ heparin, which is more heavily sulfated than heparan sulfate but can similarly block interactions between HSPGs and their targets. We found that both treatments abolished the enhanced clustering of both syn 2 and synaptophysin after $24 \mathrm{~h}$ (Fig. 4C,D). After $1 \mathrm{~h}$ of PDL bead contact, we found that heparinase treatment significantly reduced, but did not entirely abolish, the clustering of syn2 and synaptophysin (Fig. $4 E$ ). Since all the treatments were added at the same time as PDL beads, this may reflect a delay in the enzymatic activity of heparinase relative to the inductive effects of 
A

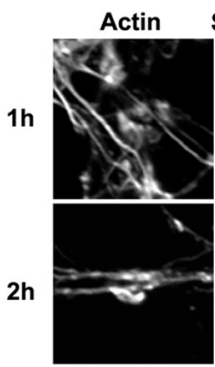

3h

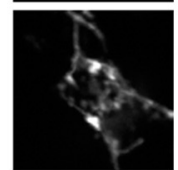

$24 h$

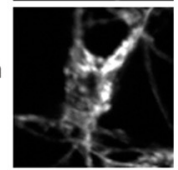

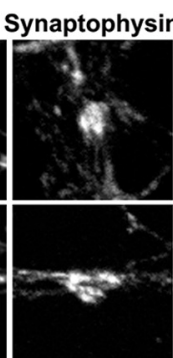
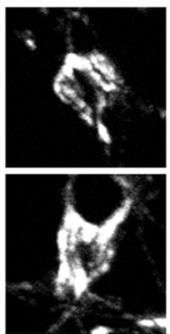

C

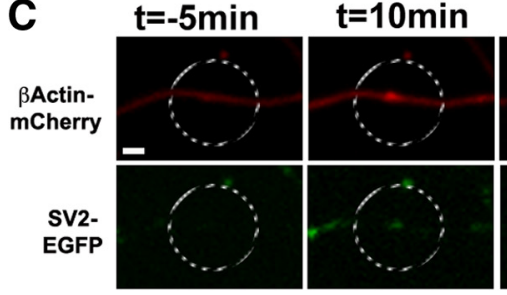

E

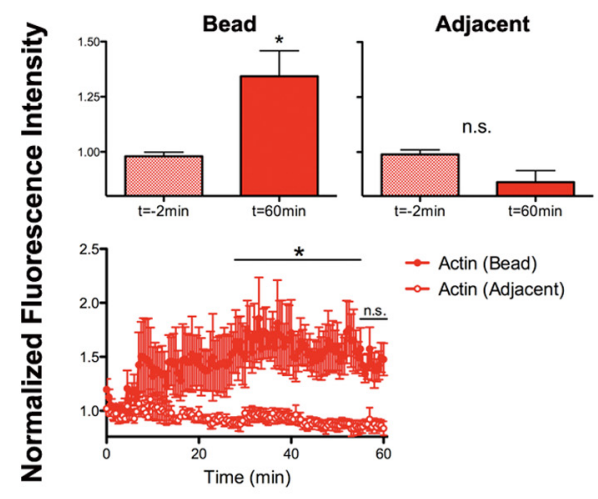

G

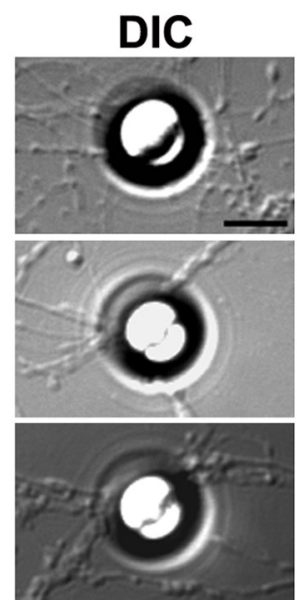

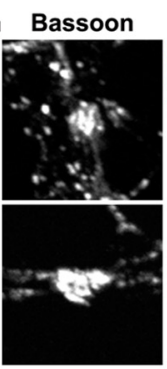
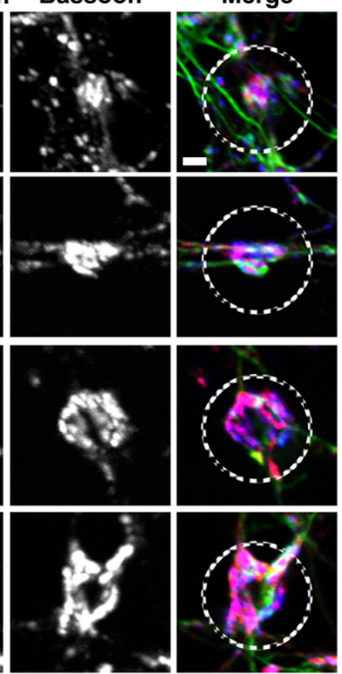

B
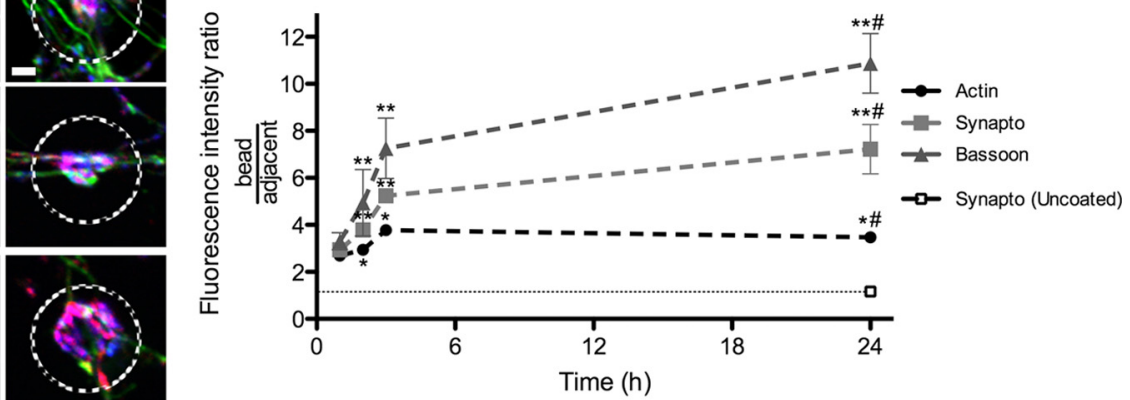

D

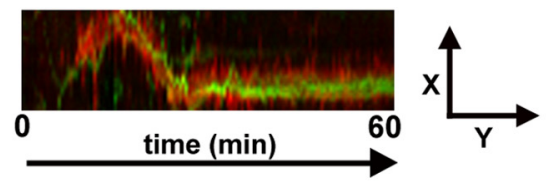

F

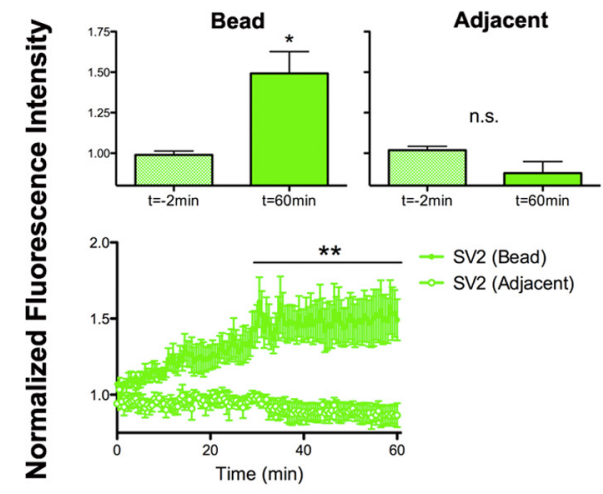

H

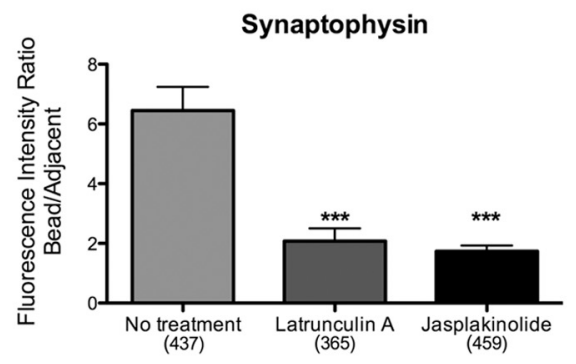

Figure 5. Coordinated recruitment of multiple presynaptic proteins to PDL bead sites proceeds by a mechanism dependent on F-actin reorganization. $A, B$, Time course study of the recruitment of actin, synaptophysin, and bassoon to PDL bead contacts. Representative images and analysis are derived from three independent experiments (DIV11-DIV13 neurons), with a minimum of 50 beads analyzed per time point per experiment. Circle, Location of the bead. $A$, Single neurite-PDL bead contacts labeled with antibodies to synaptophysin (red) and bassoon (blue) as well as Alexa-phalloidin to stain F-actin (green). Scale bar, $2 \mu \mathrm{m}$. B, Measurements of fluorescence intensity at neurite- bead contacts following incubation with PDL-coated beads $\left({ }^{*} p<0.05\right.$, ${ }^{* *} p<0.01$, $\# p>0.05$ vs 3 h). C, Individual frames collected during live time-lapse imaging of a DIV8 hippocampal culture dually infected with SV2-EGFP (green) and mCherry- $\beta$-actin (red). (Figurelegend continues.) 
the beads. However, treatment with heparin was highly effective, further reducing the PDL bead-induced clustering of both syn2 and synaptophysin after $1 \mathrm{~h}$ down to levels not significantly different from uncoated beads [syndecan-2: 1 h heparin, $1.39 \pm 0.09$ vs $24 \mathrm{~h}$ uncoated beads, $1.16 \pm 0.07$ (n.s.); synaptophysin: $1 \mathrm{~h}$ heparin, $1.35 \pm 0.07$ vs 24 h uncoated, $1.14 \pm 0.11$ (n.s.), one-way ANOVA] (Fig. 4E). Together, these results for the first time implicate syn 2 as a specific cell-surface HSPG important in triggering associated with presynapse assembly induced by PDL beads.

\section{Time-resolved determination of presynaptic protein recruitment and functionality at PDL bead sites}

A variety of recent studies show that synapses can form within a few hours of axodendritic contact both in vitro and in vivo. Therefore, our next question was, do PDL-coated beads trigger synaptic assembly in a time frame that is equivalent to that observed at native axodendritic contacts? To investigate this, we performed fixed time course studies whereby neurons were incubated with PDL-coated beads for defined time periods and triple labeled with antibodies against synaptophysin and bassoon along with Alexa-phalloidin to visualize F-actin. A rapid increase in the fluorescence intensity of all three proteins was observed within the first hour of bead contact that continued to the third hour of incubation (Fig. $5 A, B$ ). Beyond $3 \mathrm{~h}$, there was no added enhancement of fluorescence intensity of actin, while the accumulation of bassoon and synaptophysin appeared to continue, albeit modestly when compared with the $3 \mathrm{~h}$ time point (Fig. $5 A, B$ ).

Next, we used time-lapse imaging to observe the dynamics of synaptic protein recruitment to beads within the first several hours of contact. To this aim, hippocampal neurons were infected with lentivirus expressing mCherry- or EGFP-tagged synaptic proteins. In our first experiments, we focused on the axonal response of neurons by dually infecting cells with viruses expressing EGFP-tagged SV2 (EGFP-SV2) and mCherry-tagged $\beta$-actin (mCh-actin). Here, we observed a striking reorganization of $\mathrm{mCh}$-actin that occurs within the first several minutes of bead contact (Fig. 5C; supplemental Fig. S6, Movie S3, available at www.jneurosci.org as supplemental material). The mCh-actin appears very dynamic as if it is part of a filopodial-like process that is exploring the surface of the bead (see $t=30$ min time point in Fig. 5C). Intriguingly, we noticed that the accumulation of

\footnotetext{
$\leftarrow$

(Figurelegend continued.) Scale bar, $2 \mu \mathrm{m}$. D, Kymograph of the complete set of time-lapse frames from $C$, showing the relationship of actin and SV2 accumulation at the bead site. We observed a rapid and highly dynamicaccumulation of both actin and SV2 that appears to stabilize in the latter half of the time course. $\boldsymbol{E}$, Quantification and analysis of $\mathrm{mCherry}-\beta$-actin fluorescence intensity at PDL bead and adjacent control sites $(n=5)$. Top, Comparison of fluorescence intensity values taken before $(t=-2$ $\mathrm{min}$ ) and after $(t=60 \mathrm{~min})$ the addition of beads at bead (left) and adjacent (right) sites $\left({ }^{*} p<0.05\right.$, n.S., $p>0.05$, paired $t$ test). Bottom, Time course of fluorescence intensity at bead and adjacent control sites ( ${ }^{*} p<0.05$, n.S., $p>0.05$, 2-way ANOVA). $\boldsymbol{F}$, Quantification and analysis of SV2-EGFP fluorescence intensity at bead and adjacent control sites $(n=7)$. Top, Comparison of fluorescence intensity values taken before $(t=-2 \mathrm{~min})$ and after ( $t=60 \mathrm{~min})$ the addition of beads at bead (left) and adjacent (right) sites $\left({ }^{*} p<0.05 ;\right.$ n.s., $\left.p>0.05\right)$. Bottom, Time course of fluorescence intensity at bead and adjacent control sites ( ${ }^{* *} p<0.01$, 2-way ANOVA). G, H, DIV13-DIV15 hippocampal cultures were incubated with PDL-coated beads for $3 \mathrm{~h}$, either alone (no treatment) or in the presence of jasplakinolide (Jas) or latrunculin A (LatA) $(5 \mu \mathrm{M})$, which were added immediately before the addition of the beads. $\mathbf{G}$, Representative image of cultures following fixation and staining for actin and synaptophysin. Scale bar, $5 \mu \mathrm{m}$. Note the decrease in F-actin fluorescence intensity for both jasplakinolide and latrunculin $\mathrm{A}$, the former due to competition of the phalloidin agent for the same binding site as jasplakinolide, the latter due to a true loss of F-actin due to the sequestration of actin monomers. $\boldsymbol{H}$, Quantification of fluorescence intensity ratios of synaptophysin in the presence or absence of these treatments. ${ }^{* * *} p<0.001$ compared with PDL beads alone (No treatment), one-way ANOVA.
}

EGFP-SV2 is similarly rapid and appears to coincide closely with the observed reorganization of actin at bead sites (Fig. 5C, bottom) and once recruited, appeared remarkably stable throughout the remainder of the time course (Fig. 5D).

To quantify these data, we first analyzed the changes in fluorescence intensity at bead sites well as a separate, adjacent control axon for every bead site. We found a significant increase in the fluorescence intensity of both mCh-actin and EGFP-SV2 over the 60 min imaging period (Fig. $5 E, F$ ). In contrast, there was no significant accumulation of either mCh-actin or EGFP-SV2 at control nonbead sites (Fig. 5E,F).

Next, to determine the precise temporal pattern of actin and SV2 accumulation, we performed a two-way ANOVA with one repeated measure (time). For $\mathrm{mCh}$-actin, accumulation became significant at $t=28 \mathrm{~min}$ compared with the nonbead controls (Fig. 5E). This increase in $\mathrm{mCh}$-actin fluorescence intensity, although remaining elevated throughout the remainder of the time lapse, was no longer significant after $t=55 \mathrm{~min}$ (Fig. $5 E$ ), coinciding with the appearance of a stable but condensed focal site of mCh-actin (see Fig. $5 C, t=60 \mathrm{~min}$ ).

When we applied this same analysis for EGFP-SV2, we found that accumulation became significant at $t=30 \mathrm{~min}$, just $2 \mathrm{~min}$ later than mCh-actin (Fig. $5 F$ ). However, unlike mCh-actin, this increase in intensity remained significant relative to nonbead control sites for all time points after $t=30 \mathrm{~min}$ (Fig. $5 F$ ). Together, these data suggest that there is a close temporal relationship between actin dynamics and synaptic vesicle accumulation during presynapse assembly triggered by PDL-coated beads, whereby initial contact triggers a reorganization of the axonal actin cytoskeleton that is closely followed by the trapping of synaptic vesicles. To confirm that actin is not just temporally linked but is in fact critical for synaptic vesicle accumulation, we performed experiments whereby PDL-coated beads were added to hippocampal cultures (DIV13-DIV15) along with disruptors of the actin cytoskeleton and incubated for $3 \mathrm{~h}$ before fixation. At this developmental stage in vitro, it has been shown that preexisting clusters of synaptic vesicles were unaffected by actin depolymerization (Zhang and Benson, 2001). However, in the presence of (1) latrunculin A (LatA) or (2) Jasplakinolide (Jas), toxins that (1) sequester actin monomers, thereby inhibiting F-actin formation, or (2) promote the stabilization of F-actin, respectively (Spector et al., 1999), we found that synaptophysin accumulation was nearly abolished at PDL bead sites (Fig. 5G,H). These data reveal that actin reorganization is a critical step in nascent presynapse assembly, independent of neuronal age, adding to a number of studies showing that dynamic actin reorganization is an important property of axons during nascent presynapse assembly (Colicos et al., 2001; Sankaranarayanan et al., 2003; Dillon and Goda, 2005; Cingolani and Goda, 2008).

Finally, we wished to confirm that these nascent sites could recycle transmitter. To address this question, we performed experiments using cultures singly infected with EGFP-SV2, and incubated the cultures with PDL-coated beads for 1 or $24 \mathrm{~h}$. This allowed us to visualize the formation of nascent presynaptic boutons at bead sites before stimulation, and to select for bead sites containing SV clusters. We then monitored the ability of these boutons to recycle the vital dye FM4-64. After $1 \mathrm{~h}$ of incubation with PDL-coated beads, we observed bright EGFP-SV2-positive clusters outlining the bead perimeter at a subset of bead contacts, in both young (supplemental Fig. S5A, available at www.jneurosci. org as supplemental material) and more mature (supplemental Fig. S5E, available at www.jneurosci.org as supplemental material) cultures. After $24 \mathrm{~h}$, most bead contacts contained multiple 
A

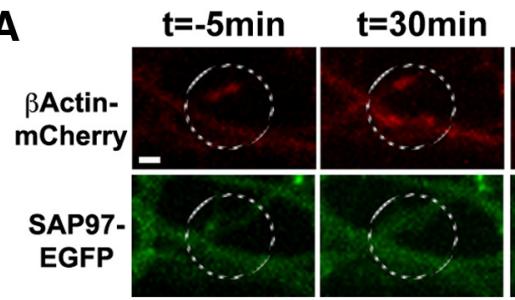

c
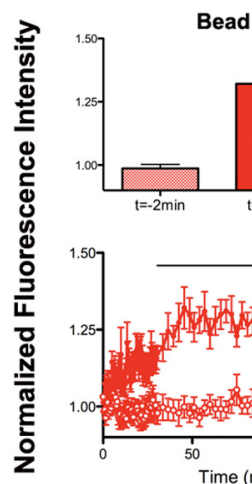

Bead
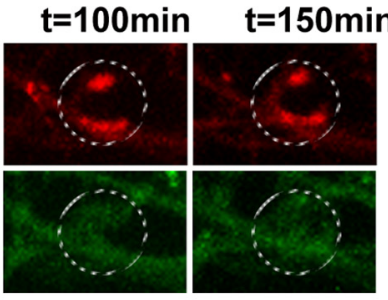

Adjacent
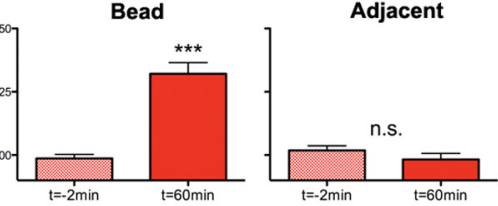

$\rightarrow$ Actin (Bead)

$\rightarrow$ Actin (Adjacent)

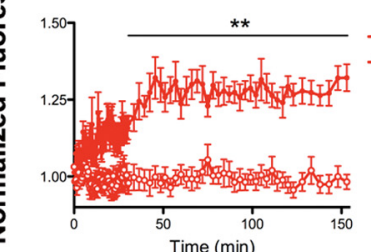

$\mathbf{E}$
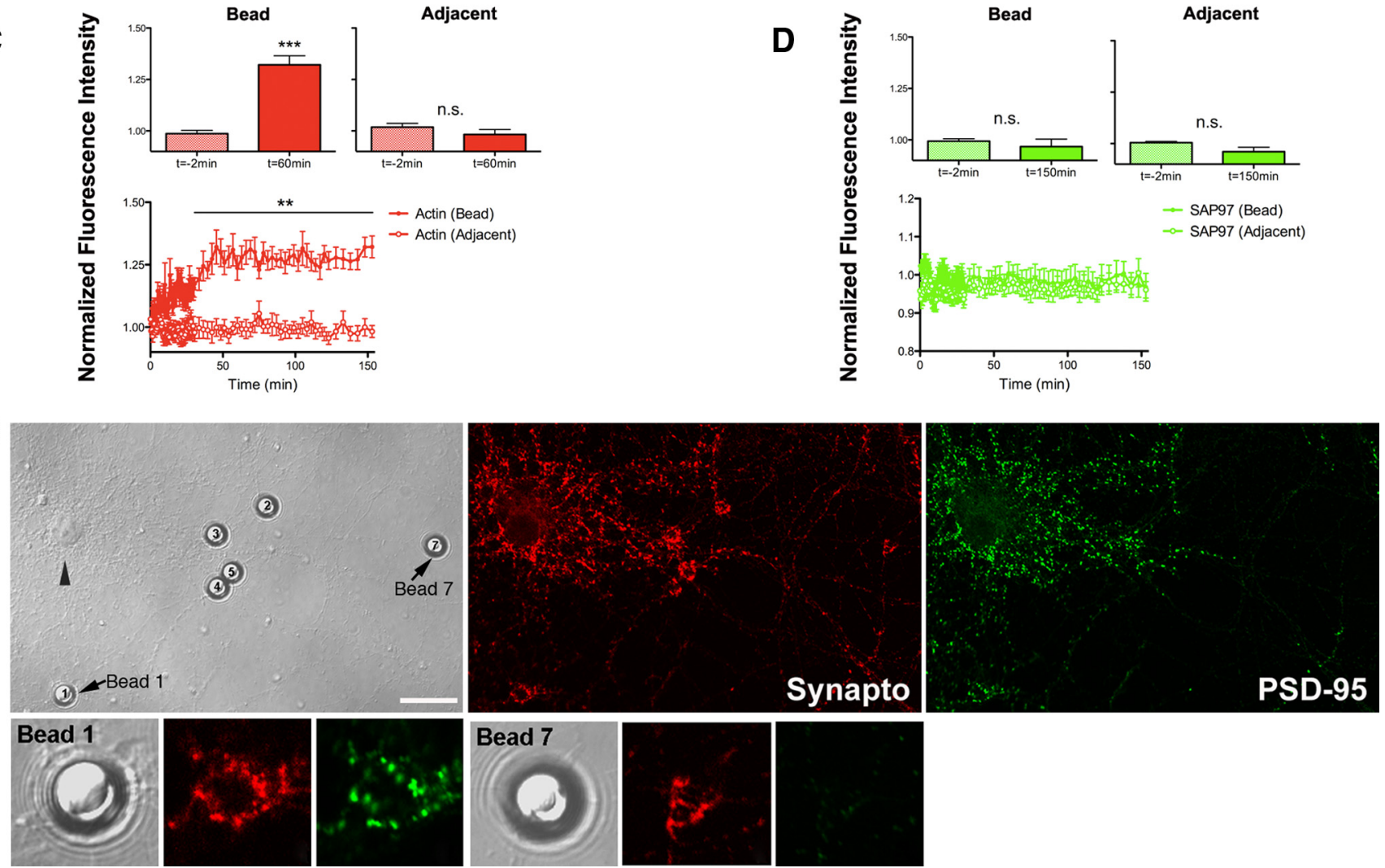

Figure 6. PDL-coated beads can facilitate postsynaptic differentiation on beads where presynaptic clusters are also observed. $\boldsymbol{A}$, Individual frames collected during live time-lapse imaging of dendrites from DIV13 hippocampal neurons dually infected with SAP97-EGFP (green) and mCh-actin (red). Circle, Location of the bead. Scale bar, $2 \mu \mathrm{m}$. $\boldsymbol{B}$, Kymograph of the complete set of time-lapse frames from $A$, showing the relationship of $\mathrm{mCh}$-actin and SAP97 accumulation at the bead site. We observe that actin accumulates at the bead site, while SAP97 fluorescence does not appear to change. $C$, Quantification and analysis of $\mathrm{mCh}$-actin fluorescence intensity at bead and adjacent control sites $(n=9)$. Top, Comparison of fluorescence intensity values taken before ( $t=$ $-2 \mathrm{~min})$ and after $(t=150 \mathrm{~min})$ the addition of beads at bead (left) and adjacent (right) sites (*** $p<0.001$, n.s., $p>0.05)$. Bottom, Time course of fluorescence intensity at bead and adjacent control sites $\left({ }^{* *} p<0.01\right.$, 2-way ANOVA). D, Quantification and analysis of SAP97-EGFP fluorescence intensity at bead and adjacent control sites $(n=9)$. Top, comparison of fluorescence intensity values taken before $(t=-2 \mathrm{~min})$ and after ( $t=150 \mathrm{~min}$ ) addition of beads at bead (left) and adjacent (right) sites ( $p>0.05)$. Bottom, Time course of fluorescence intensity at bead and adjacent control sites. No significant changes in fluorescence intensity were observed at any time point. $E$, Representative image of a DIV15 neuron cocultured with beads for $24 \mathrm{~h}$ (DIC) and immunostained for synaptophysin (red) and PSD95 (green). In the upper left DIC image, the beads are numbered to demarcate their proximity relative to the cell body (arrowhead). Bottom, Two neurite-PDL bead contacts, one of which exhibits numerous synaptophysin as well as PSD95 clusters (bead 1) and the other showing synaptophysin clustering alone (bead 7). Scale bar, $20 \mu \mathrm{m}$.

EGFP-SV2-positive clusters (supplemental Fig. S5C,G, available at www.jneurosci.org as supplemental material). When stimulated by a high- $\mathrm{K}^{+}$solution in the presence of the FM dye, we observed robust uptake of the FM dye at these sites (supplemental Fig. S5 A, C, E, G, $1 \times \mathrm{KCl}$ panel, available at www.jneurosci.org as supplemental material) that was unloaded following a second stimulation (supplemental Fig. S5 A, C, E, G, $2 \times \mathrm{KCl}$ panel, available at www.jneurosci.org as supplemental material). Following quantification, we found that the unloading of the FM dye was significant at all time points tested (supplemental Fig. S5B,D,F,H, available at www.jneurosci.org as supplemental material). These results confirm that presynaptic functionality can be achieved rapidly at nascent presynaptic endings formed at PDL bead contacts, as has been observed between native contacts in situ (Ahmari et al., 2000; Friedman et al., 2000).
PDL-coated beads facilitate postsynaptic differentiation that is delayed and dependent on the presence of presynaptic clusters

It is clear both from previous work and our data that substratebound polylysine can induce presynaptic assembly; however, whether these beads can induce postsynaptic assembly in central neurons is unclear. To investigate the capacity of PDL beads to drive postsynaptic development, we first performed live imaging studies using cultures dually infected with the postsynaptic protein SAP97-EGFP along with mCh-actin. By visual inspection, the PDL beads adhered to dendrites with similar speed and resistance to dislodgment as was observed along axons. Furthermore, similar to our observations of PDL beads contacting axons, we also observed an accumulation of $\mathrm{mCh}$-actin at sites of dendritic contact (Fig. 6A, B; supplemental Fig. S6, Movie S4, available at 
Table 2. Quantification and analysis of PSD95-synaptophysin dual labeling experiments

\begin{tabular}{|c|c|c|c|c|c|c|c|c|c|}
\hline & \multicolumn{2}{|l|}{ Total } & \multicolumn{2}{|c|}{ Both enhanced } & \multicolumn{2}{|c|}{ Just synaptophysin } & \multicolumn{2}{|l|}{ Just PSD95 } & \multirow[b]{2}{*}{ Neither } \\
\hline & Synapto & PSD95 & Synapto & PSD95 & Synapto & PSD95 & Synapto & PSD95 & \\
\hline Fluorescence intensity ratio (mean \pm SEM) & $14.7 \pm 1.9$ & $3.4 \pm 0.5$ & $17.1 \pm 2.7$ & $7.5 \pm 1.2$ & $17.5 \pm 3.5$ & $0.8 \pm 0.1$ & $1.6 \pm 0.1$ & $2.9 \pm 0.4$ & \\
\hline Number of beads & 207 & & 79 & & 94 & & 3 & & 31 \\
\hline Proportion & $100 \%$ & & $38.2 \%$ & & $45.4 \%$ & & $1.4 \%$ & & $15 \%$ \\
\hline
\end{tabular}

The fluorescence ratios for both PSD95 and synaptophysin were quantified and binned according to whether a single bead site had a more than twofold enhancement of PSD95, synaptophysin, or both. The pooled fluorescence ratios are also shown for each category.

www.jneurosci.org as supplemental material). In contrast, there was no net change in $\mathrm{mCh}$-Actin fluorescence intensity at adjacent control sites (Fig. 6C). When analyzed by two-way ANOVA, this dendritic accumulation of $\mathrm{mCh}$-actin appeared to follow a similar time course as axonal mCh-actin, as the accumulation of actin became significant at $t=30.5 \mathrm{~min}$ and remained so throughout the remainder of the time course (Fig. 6C). However, we did not observe any accumulation of SAP97-EGFP-positive puncta at any time during imaging, even after several hours (Fig. $6 A, B, D$; supplemental Movie S4, available at www.jneurosci.org as supplemental material). These latter results show that while beads can adhere to dendrites, and can induce actin reorganization that appears to be similar to that seen along axons, this does not lead to a postsynaptic differentiation on a time frame in which presynaptic differentiation may be observed along axons.

We hypothesized that perhaps postsynaptic protein accumulation proceeds in response to PDL beads, but is simply delayed relative to presynaptic differentiation, as has been reported previously (Friedman et al., 2000; Okabe et al., 2001). To address this question, we next performed coimmunofluorescence experiments of synaptophysin with PSD95, a marker of the postsynaptic density, following $24 \mathrm{~h}$ of coculture of DIV15 neurons with PDL beads. When we quantified this data we found that, like synaptophysin, PSD95 immunofluorescence is also enhanced at PDL bead sites, albeit to a significantly lower degree (Fig. 6E, Table 2). Closer inspection of the data, however, revealed some interesting trends.

Where beads contacted few thin-diameter processes located distally to the cell body, indicative of axons, it appeared that only synaptophysin clustering is induced (Fig. 6E, bead 7). When beads contacted neurites closer to the cell body, presumably where there is a higher density of dendrites, PSD95 clusters were also observed (Fig. 6E, bead 1). To investigate this further, we classified the fluorescence intensity ratios into the following categories: where both synaptophysin and PSD95 were increased more than twofold, where synaptophysin alone or PSD95 alone were increased more than twofold, and where neither were increased. We found that $\sim 38 \%$ of the contacts displayed dually enhanced synaptophysin and PSD95 labeling, but an even greater proportion (45.4\%) of the contacts displayed enhanced synaptophysin clustering in the absence of enhanced PSD95 clustering (Table 2). In contrast, the reverse (enhanced PSD95 without enhanced synaptophysin) virtually never happened (3/207 PDLcoated beads) (Table 2). These results suggest that perhaps postsynaptic differentiation may indeed be induced at sites of bead contact, but depends on the presence of differentiated presynaptic boutons.

Interdependence of presynaptic and postsynaptic elements on cognate synaptic development

Previous studies have shown that the induction of presynaptic or postsynaptic differentiation can be achieved by the expression of subsets of synaptic adhesion molecules such as neuroligin, syn-
CAM, or neurexin on the surface of heterologous cells in the place of native neuronal membranes (Scheiffele et al., 2000; Biederer et al., 2002; Graf et al., 2004; Sara et al., 2005; Kim et al., 2006). These molecules are part of a growing family of synaptic recognition molecules that are thought to behave as the earliest molecular triggers of synaptic development. However, the beads used in the present study were not coated with any of these or other native molecules shown in vitro to drive presynaptic or postsynaptic assembly, suggesting that PDL-coated beads bypass these cognate recognition cues. To rule out the possibility that PDL bead-induced presynapse assembly relied on cues derived from nearby or contacting postsynaptic membranes, we grew hippocampal neurons on a micropatterned substrate, which facilitates the separation of axons from dendrites by encouraging growth along defined linear pathways. In these cultures, only isolated taul-positive axons contacting PDL-coated beads displayed robust synaptophysin clustering, while beads contacting isolated MAP2-positive dendrites did not (Fig. $7 A, B$ ). This result confirms that PDL-coated beads exclusively induce SV clustering on axons and do not require the presence of dendritic membranes to do so.

The intriguing converse question to the above experiments is whether isolated postsynaptic boutons could be induced to form by PDL-coated beads, or whether differentiation of a postsynapse is dependent on presynapse assembly. Although it is generally difficult to separate axons from dendrites at stages of neuronal differentiation when postsynaptic protein clusters are forming (DIV9 and beyond), we were able to observe several examples of beads contacting isolated dendrites expressing SAP97-EGFP. After $1 \mathrm{~h}$ of incubation with PDL-coated beads, we did not observe appreciable clustering of SAP97-EGFP at sites of bead contact (Fig. 7C, circles), consistent with our time-lapse imaging data (Fig. 6A-D). After $24 \mathrm{~h}$ of bead contact, we did observe robust clustering of SAP97-EGFP only at sites where beads contacted several processes, presumably mixed tracts of axons and dendrites (Fig. 7D, arrowheads). These data are consistent with our previous PSD95 data (Fig. 6E). However, even at this $24 \mathrm{~h}$ time point, there was no enhanced clustering at bead sites contacting isolated SAP97-positive processes (Fig. 7D, circles). Together, these results clearly show that PDL-coated beads possess the capacity to bypass native cognate interactions that normally trigger presynaptic development, effectively substituting for the postsynaptic ending. In contrast, this type of interaction between bead and dendritic membrane is insufficient to drive postsynaptic assembly, and lends strength to our assertion that postsynaptic development observed at bead sites is not driven by the bead but by the presence of the presynaptic ending.

\section{Discussion}

This work broadly extends some early observations revealing that axons have the remarkable capacity to form presynaptic compartments onto artificial substrates. By EM, prior studies characterized the SV clustering phenomenon in detail (Burry, 1980, 
1982, 1983, 1985; Peng et al., 1987). Because substrates were necessarily polybasic for the clustering effect to occur, it was concluded that electrostatic-based adhesion between an axon and its target represents the first step that triggers synapse assembly (Burry, 1980). While intriguing, these studies did not sufficiently define the early assembly steps to place adhesion within a logical sequence of events. Furthermore, it was never clear how these artificial substrates interacted with neuronal membranes to trigger SV clustering. Finally, these studies did not determine whether the observed SV clusters represented true presynaptic boutons, nor did they define the potential of these substrates to induce postsynaptic development. In the present work, we sought to revive an old experimental approach to reveal novel facets of the synaptogenic process. Our most significant findings are as follows.

\section{Adhesion as a first step}

First, we confirm that adhesion rather than synaptic protein clustering is indeed the first step toward building a functional, stable presynapse. Using a combination of live imaging and atomic force microscopy, we show that PDL-coated beads are resistant to detachment well in advance of any synaptic protein accumulation. We find that SV clusters along axons are highly mobile unless and until they encounter a bead, shortly after which they stabilize and intensify with time. Furthermore, SV clusters could be recruited to bead sites placed anywhere along the length of the axon, suggesting that synapse assembly in situ does not necessarily take place at sites of preexisting presynaptic clusters, but rather relies on target adhesion to trigger synaptic protein recruitment.

\section{Role of charge distribution and recruitment of endogenous factors}

Our observations distinguish not just charge (Burry, 1980) but presentation of charge as critical for triggering presynapse assembly. We find that cationic polypeptides immobilized on the bead surface selectively provide a platform that is both adhesive and synaptically inductive, while positively charged lipids do not. In addition, we identify HSPGs, including the membrane spanning HSPG syndecan-2, as an important class of ECM molecules driving PDL beadinduced synapse formation. HSPGs are a family of molecules with enormous structural diversity and thus are rather intimidating to study. Yet, their ubiquitous expression, anionic structure, and
A
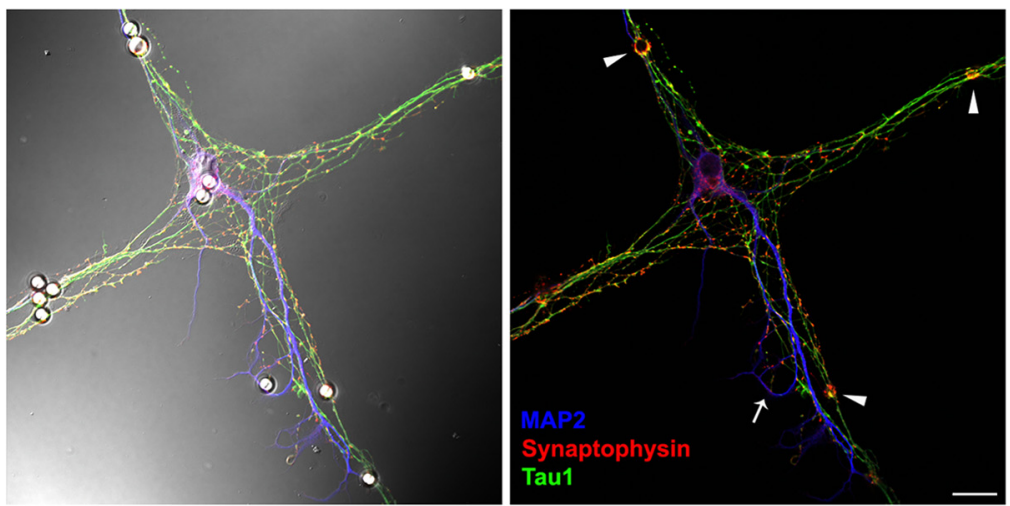

B
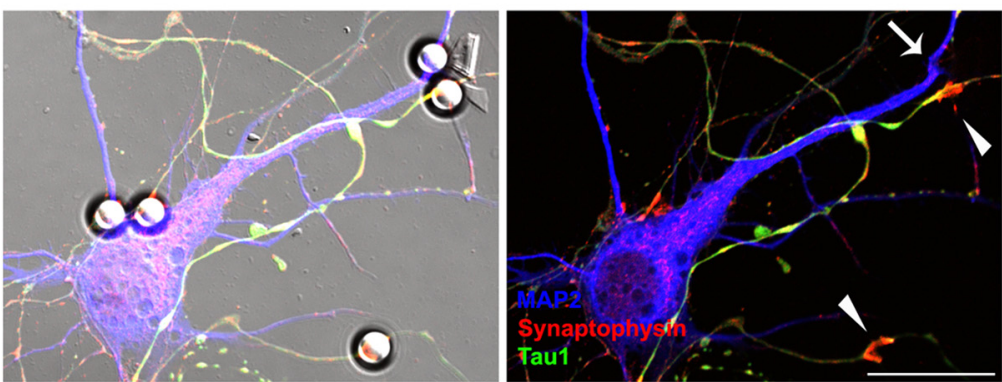

C

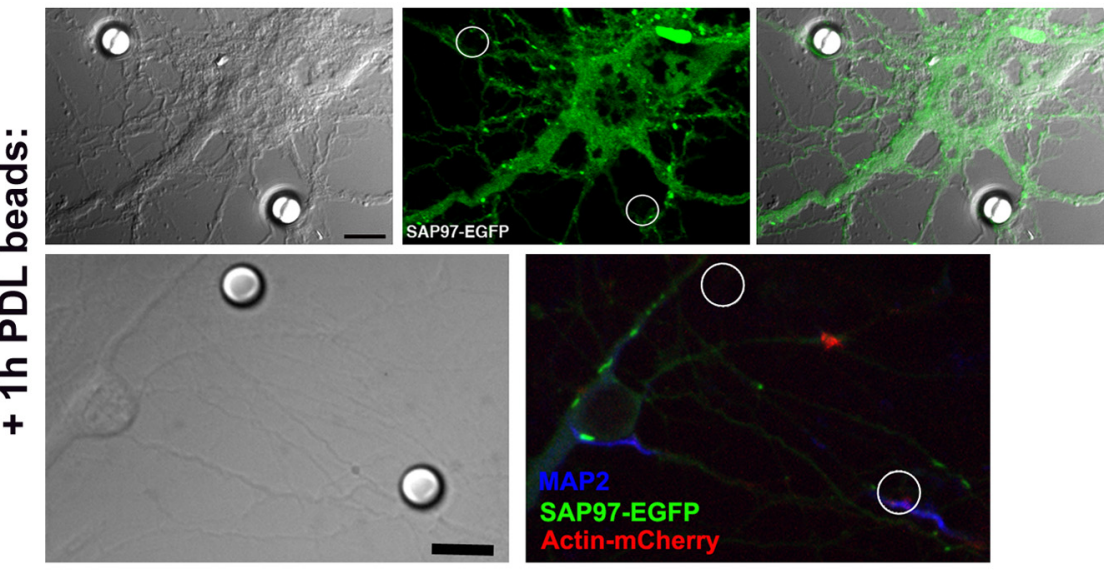

D

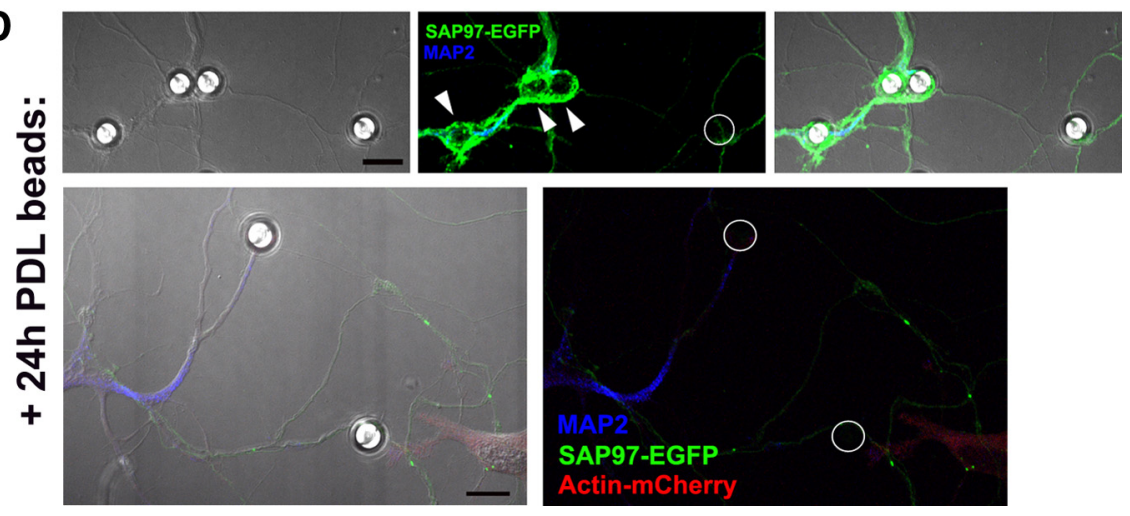

Figure 7. Dependency of presynaptic development on postsynaptic assembly and of postsynaptic development on presynaptic assembly. $\boldsymbol{A}, \boldsymbol{B}$, Representative images of DIV7 neurons cultured on a micropatterned substrate and triple stained for Tau 1 (green), MAP2 (blue), and synaptophysin (red) following incubation with PDL-coated beads for $24 \mathrm{~h}$. Notice the separation of axons from dendrites and the selective enhancement of synaptophysin exclusively on axons (arrowheads) and not on dendrites (arrows). Left-hand images are the merged fluorescence and DIC images to denote the location of the beads. Scale bars, $20 \mu \mathrm{m}$. $\boldsymbol{C}, \boldsymbol{D}$, Representative confocal images of DIV9, SAP97-EGFP-infected cultures (green) incubated with PDL-coated beads. Circles and arrowheads denote the location of beads shown in corresponding DIC (right) and/or merge (left) images. Scale bars, $10 \mu \mathrm{m}$. C, DIV9 cultures $+1 \mathrm{~h}$ PDL beads. Top, Singly infected SAP97-EGFP culture. Bottom, Dually infected SAP97-EGFP/mCh-actin (red) culture immunostained for MAP2 (blue). D, DIV9 cultures $+24 \mathrm{~h}$ PDL beads. Top, Singly infected SAP97-EGFP (green) culture immunostained for MAP2 (blue). Bottom, Dually infected SAP97-EGFP/mCh-actin (red) culture immunostained for MAP2 (blue). 
A
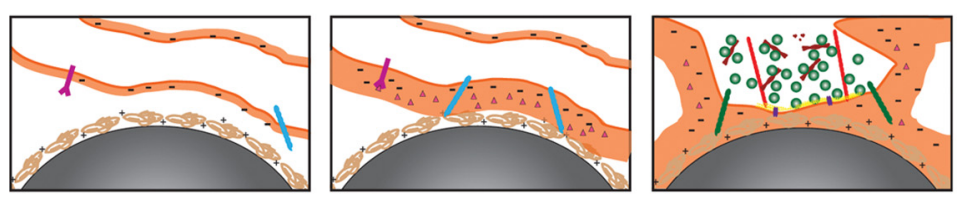

B

Axon-bead contact:
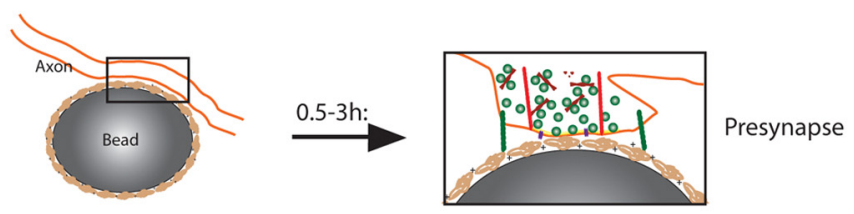

Dendrite-bead contact:
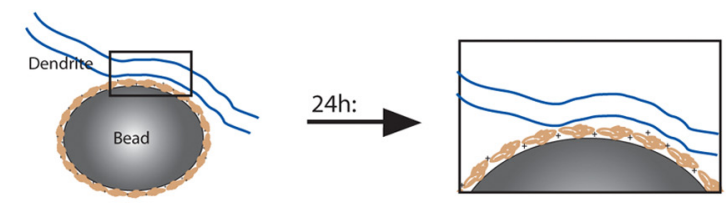

No postsynapse

Axon-dendrite -bead contact:
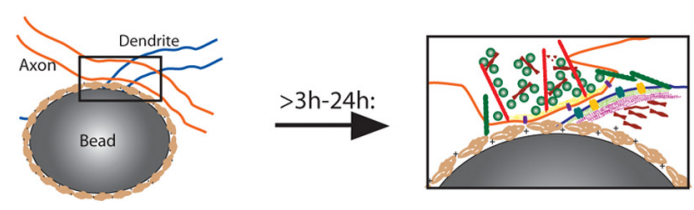

Presynapse then postsynapse

Figure 8. A, Model to describe the function of the ECM in PDL bead-induced presynapse formation. The ECM is rich in a variety of heavily charged and complex molecules, including HSPGs, that forms a scaffold for the heterophilic binding of soluble and transmembrane proteins. The binding of a PDL bead to an axon (left) could therefore encourage (1) the heterophilic binding of transmembrane HSPGs, including syn2 and potentially other cell-surface receptors, (2) the presentation of soluble synaptogenic ligands to the axon, or both (middle). This results in the triggering of signaling mechanisms resulting in the assembly of a presynapse (right). $\boldsymbol{B}$, Model for distinguishing the responsiveness of axons versus dendrites to form synaptic-like contacts to PDL beads. Top, Upon contact with a PDL-coated bead, an axon isolated from any native targets will rapidly form a functional presynaptic ending. In contrast, a bead contacting an isolated dendrite (middle) will adhere, but not trigger the formation of a postsynaptic density even after $24 \mathrm{~h}$. Bottom, A PDL bead making simultaneous contact with an axon and a dendrite can encourage the formation of a native synapse that, given the inability of the PSD to form on isolated dendrites, is likely driven by the presynaptic bouton induced to form by the bead.

functional excitatory or inhibitory presynapses. These bead-presynaptic complexes are highly stable, unlike the mobile presynaptic-like clusters observed along isolated axons (Krueger et al., 2003). They contain presynaptic proteins in their correct dispositions, depend on F-actin reorganization for their assembly, and are capable of the uptake and release of the styryl dye FM4-64 as quickly as within $1 \mathrm{~h}$ following PDL bead contact. When considered in the context of other studies on presynaptic development (Ahmari et al., 2000; Friedman et al., 2000; Jontes et al., 2000; Shapira et al., 2003; Tsuriel et al., 2006), these observations strongly suggest that the assembly process triggered by PDLcoated beads is highly similar to that which occurs in situ.

\section{Postsynaptic development only driven by the nascent presynapse}

In contrast to the readiness by which the presynaptic ending forms to the bead, the bead does not act as a postsynaptic inducer (Fig. $8 \mathrm{~B}$ ). Where postsynaptic differentiation was observed, it was nearly always in relation to already formed presynaptic structures on the bead, suggesting that the nascent presynapse is critical in directing postsynaptic differentiation in this system (Fig. $8 B$ ). The mechanism by which this takes place is unclear but could involve the encouragement of selec-

capacity to act as critical coreceptors for several growth factor molecules make them uniquely poised to participate in a number of cell biological processes. In the CNS, this family of molecules is becoming increasingly linked to diverse neurodevelopmental processes including neuronal proliferation, differentiation, and neurite outgrowth (Bandtlow and Zimmermann, 2000; Kleene and Schachner, 2004; de Wit and Verhaagen, 2007). In the present study, we further highlight their role in synaptogenic processes by identifying them as critical mediators in PDL beadinduced synapse assembly and identify a specific cell surface molecule, syndecan2, as at least one important molecular subtype involved in these effects. Furthermore, the structure and localization of syn 2 at the cell surface suggests a mechanism whereby the bead binds directly to axonal membranes and their receptors, thereby activating signaling processes that lead to presynapse assembly (Fig. $8 A$ ). Yet, at the moment we cannot rule out the possibility that other cell surface or secreted HSPG subtypes are also involved, possibly by adsorbing onto the bead surface and further recruiting other necessary soluble components (Fig. $8 \mathrm{~A}$ ). It will be of great interest in the future to identify other molecular mediators that can interact in a heterophilic manner with PDL beads. Nevertheless, these findings underscore an important role for the ECM as an adhesive support and a scaffolding structure that can promote synaptogenesis.

\section{Presynaptic complexes are true boutons, not mere synaptic vesicle clusters}

The ensuing presynaptic specializations formed between neuronal processes and PDL-coated beads are more than simple SVfilled varicosities (Burry, 1980, 1982; Peng et al., 1987), but tive interactions between cognate presynaptic and postsynaptic molecules beneath the bead, a fitting explanation given that in other studies of postsynapse formation in vitro, the presynaptic membrane could only be replaced by endogenous synaptogenic inducers (Graf et al., 2004; Kim et al., 2006; Ko et al., 2006). Although synaptogenic inducers derived from postsynaptic membranes can also drive presynaptic development (Scheiffele et al., 2000; Biederer et al., 2002; Dean et al., 2003; Kayser et al., 2006; Dalva et al., 2007), our system shows that presynapse formation can proceed in the absence of the postsynaptic membrane on which they are expressed.

\section{Permissive versus inductive: what is necessary to trigger the formation of a synaptic ending?}

Following his groundbreaking observations that transected optic nerve axons can regrow to their target destinations in the tectum, Sperry (1963) hypothesized that the establishment of neuronal circuitry is critically dependent on the expression of selective cytochemical markers at the appropriate location and concentration. In the context of synapse formation, a growing consensus in the field is that cell adhesion molecules (CAMs), several classes of which are enriched at central synapses (Gerrow et al., 2006; Dalva et al., 2007; Shapiro et al., 2007), act as the molecular tags that correctly identify synaptic partners to each other, and provide the adhesive substrate to lock in synaptic membranes. Other molecules also demonstrated to be synapse inducing fall into a variety of categories, including soluble growth factors (Hall et al., 2000; Withers et al., 2000; Umemori et al., 2004; Ledda et al., 2007), extracellular matrix factors (Noakes et al., 1995; Gautam et al., 
1996; Fox et al., 2007; Bogdanik et al., 2008; Sato et al., 2008), and astrocyte-derived molecules (Ullian et al., 2001; Christopherson et al., 2005).

The molecular diversity and selective interactions of these molecules satisfies the specificity that is required in accordance with Sperry's hypothesis. However, one must address the concept of synaptic molecules being permissive versus those that are truly inductive, a controversy that arises when one examines the published data focused on certain adhesion molecules. Despite clear evidence that they can induce synaptogenesis in vitro (Scheiffele et al., 2000; Biederer et al., 2002; Dean et al., 2003; Graf et al., 2004; Kayser et al., 2006), and that altering their expression levels alters the number of synapses in vitro (Ethell et al., 2001; Chih et al., 2005; Sara et al., 2005), their genetic deletion in vivo does not dissolve synaptogenesis (Henkemeyer et al., 2003; Missler et al., 2003; Fujita et al., 2006; van der Weyden et al., 2006; Varoqueaux et al., 2006; Dudanova et al., 2007). This would suggest that in vivo, synaptic CAMs most critically function at later stages of maturation rather than synaptic induction.

Another issue relates to whether or not synaptic inducers act at within defined time frames during nascent synaptogenesis. This has been raised in a recent study at the NMJ, in which it was shown that agrin, FGF, laminin- $\beta 2$, and distinct collagen IV chains, which could all be classified as synaptic inducers, were expressed at discrete times during development and regulated distinct aspects of synapse formation in situ (Fox et al., 2007). Thus, a major challenge in the field will be to classify chemotactic synaptogenic molecules in accordance with the complexity present within the nascent synaptic environment.

Finally, given the extraordinary production of synapses during the late gestational and early postnatal periods of mammalian brain development, many of which are eliminated at later stages, it has been hypothesized that initial synaptic connections lack precision and refinement of preexisting connections that are initially formed promiscuously lead to the establishment of mature neuronal circuits (Changeux and Danchin, 1976; Katz and Shatz, 1996; Webb et al., 2001; Jontes and Phillips, 2006; Zhang, 2006). These in vivo observations have not been complemented by current cellular models and yet if true, suggest that a high degree of specificity is not required to trigger synapses to form in the earliest stages.

In our system, the surprising ease with which the presynaptic compartment self-assembles underscores the underestimated capacity for neurons to form rather indiscriminate synaptic connections. Our observations are unlikely to be an artifact of culture, but support a large body of literature demonstrating that axons possess the capacity to drive synaptogenesis following contact with a variety of unnatural targets (Schacher et al., 1985; Sur et al., 1988; Haydon and Zoran, 1989; Zwimpfer et al., 1992), some of which were shown to be functional in behavioral assays (Jacobson and Baker, 1968). While the identification of cellsurface and possibly secreted HSPGs does suggest that the bead requires certain endogenous, and thus specific, factors to drive presynapse assembly, we nevertheless conclude that point-topoint specificity between natural targets is not necessarily required. Instead, such specificity could arise during later stages of synaptogenesis, such as following strengthening by activity- and experience-dependent mechanisms (Changeux and Danchin, 1976; Jontes and Phillips, 2006). A developmental strategy whereby more generalized requirements are needed to form synapses could ensure that sufficient numbers of synapses are formed during development, when many more synapses are formed than retained en route to the formation of mature neuronal circuits (Katz and Shatz, 1996).

\section{Conclusions}

In the present study, we have shown that a simple system of cultured neurons and PDL-coated beads can be ideally suited to probe the earliest events by which nascent synapses form. This system, which can define the temporal appearance of synaptic molecules with precise spatial control, will likely be useful in many types of studies of synaptic development.

\section{References}

Ahmari SE, Buchanan J, Smith SJ (2000) Assembly of presynaptic active zones from cytoplasmic transport packets. Nat Neurosci 3:445-451.

Bandtlow CE, Zimmermann DR (2000) Proteoglycans in the developing brain: new conceptual insights for old proteins. Physiol Rev 80:12671290.

Banker G, Goslin K (1998) Culturing nerve cells, Ed 2. Cambridge, MA: MIT.

Benson DL, Tanaka H (1998) N-Cadherin redistribution during synaptogenesis in hippocampal neurons. J Neurosci 18:6892-6904.

Biederer T, Sara Y, Mozhayeva M, Atasoy D, Liu X, Kavalali ET, Südhof TC (2002) SynCAM, a synaptic adhesion molecule that drives synapse assembly. Science 297:1525-1531.

Bogdanik L, Framery B, Frölich A, Franco B, Mornet D, Bockaert J, Sigrist SJ, Grau Y, Parmentier ML (2008) Muscle dystroglycan organizes the postsynapse and regulates presynaptic neurotransmitter release at the Drosophila neuromuscular junction. PLoS One 3:e2084.

Burry RW (1980) Formation of apparent presynaptic elements in response to poly-basic compounds. Brain Res 184:85-98.

Burry RW (1982) Development of apparent presynaptic elements formed in response to polylysine coated surfaces. Brain Res 247:1-16.

Burry RW (1983) Postnatal rat neurons form apparent presynaptic elements on polylysine-coated beads in vivo. Brain Res 278:236-239.

Burry RW (1985) Protein synthesis requirement for the formation of synaptic elements. Brain Res 344:109-119.

Cardin AD, Weintraub HJ (1989) Molecular modeling of proteinglycosaminoglycan interactions. Arteriosclerosis 9:21-32.

Chang HC, Samaniego F, Nair BC, Buonaguro L, Ensoli B (1997) HIV-1 Tat protein exits from cells via a leaderless secretory pathway and binds to extracellular matrix-associated heparan sulfate proteoglycans through its basic region. AIDS 11:1421-1431.

Changeux JP, Danchin A (1976) Selective stabilization of developing synapses as a mechanism for the specification of neuronal networks. Nature 264:705-712.

Chih B, Engelman H, Scheiffele P (2005) Control of excitatory and inhibitory synapse formation by neuroligins. Science 307:1324-1328.

Christopherson KS, Ullian EM, Stokes CC, Mullowney CE, Hell JW, Agah A, Lawler J, Mosher DF, Bornstein P, Barres BA (2005) Thrombospondins are astrocyte-secreted proteins that promote CNS synaptogenesis. Cell 120:421-433.

Cingolani LA, Goda Y (2008) Actin in action: the interplay between the actin cytoskeleton and synaptic efficacy. Nat Rev Neurosci 9:344-356.

Colicos MA, Collins BE, Sailor MJ, Goda Y (2001) Remodeling of synaptic actin induced by photoconductive stimulation. Cell 107:605-616.

Dalva MB, McClelland AC, Kayser MS (2007) Cell adhesion molecules: signaling functions at the synapse. Nat Rev Neurosci 8:206-220.

Dean C, Scholl FG, Choih J, DeMaria S, Berger J, Isacoff E, Scheiffele P (2003) Neurexin mediates the assembly of presynaptic terminals. Nat Neurosci 6:708-716.

de Wit J, Verhaagen J (2007) Proteoglycans as modulators of axon guidance cue function. In: Semaphorins: receptor and intracellular signaling mechanisms (Pasterkamp RJ, ed), pp 73-89. Berlin: Springer.

Dillon C, Goda Y (2005) The actin cytoskeleton: integrating form and function at the synapse. Annu Rev Neurosci 28:25-55.

Dityatev A, Schachner M (2006) The extracellular matrix and synapses. Cell Tissue Res 326:647-654.

Dityatev A, Dityateva G, Sytnyk V, Delling M, Toni N, Nikonenko I, Muller D, Schachner M (2004) Polysialylated neural cell adhesion molecule promotes remodeling and formation of hippocampal synapses. J Neurosci 24:9372-9382. 
Dow KE, Mirski SE, Roder JC, Riopelle RJ (1988) Neuronal proteoglycans: biosynthesis and functional interaction with neurons in vitro. J Neurosci $8: 3278-3289$.

Dudanova I, Tabuchi K, Rohlmann A, Südhof TC, Missler M (2007) Deletion of alpha-neurexins does not cause a major impairment of axonal pathfinding or synapse formation. J Comp Neurol 502:261-274.

Ethell IM, Yamaguchi Y (1999) Cell surface heparan sulfate proteoglycan syndecan-2 induces the maturation of dendritic spines in rat hippocampal neurons. J Cell Biol 144:575-586.

Ethell IM, Irie F, Kalo MS, Couchman JR, Pasquale EB, Yamaguchi Y (2001) EphB/Syndecan-2 signaling in dendritic spine morphogenesis. Neuron 31:1001-1013.

Fannon AM, Colman DR (1996) A model for central synaptic junctional complex formation based on the differential adhesive specificities of the cadherins. Neuron 17:423-434.

Fox MA, Sanes JR, Borza D-B, Eswarakumar VP, Fässler R, Hudson BG, John SWM, Ninomiya Y, Pedchenko V, Pfaff SL, Rheault MN, Sado Y, Segal Y, Werle MJ, Umemori H (2007) Distinct target-derived signals organize formation, maturation, and maintenance of motor nerve terminals. Cell 129:179-193.

Friedman HV, Bresler T, Garner CC, Ziv NE (2000) Assembly of new individual excitatory synapses: time course and temporal order of synaptic molecule recruitment. Neuron 27:57-69.

Fujita E, Kouroku Y, Ozeki S, Tanabe Y, Toyama Y, Maekawa M, Kojima N, Senoo H, Toshimori K, Momoi T (2006) Oligo-astheno-teratozoospermia in mice lacking RA175/TSLC1/SynCAM/IGSF4A, a cell adhesion molecule in the immunoglobulin superfamily. Mol Cell Biol 26:718-726.

Gallagher JT, Lyon M, Steward WP (1986) Structure and function of heparan sulfate proteoglycans. Biochem J 236:313-325.

Gautam M, Noakes PG, Moscoso L, Rupp F, Scheller RH, Merlie JP, Sanes JR (1996) Defective neuromuscular synaptogenesis in agrin-deficient mutant mice. Cell 85:525-535.

Gerrow K, Romorini S, Nabi SM, Colicos MA, Sala C, El-Husseini A (2006) A preformed complex of postsynaptic proteins is involved in excitatory synapse development. Neuron 49:547-562.

Graf ER, Zhang X, Jin SX, Linhoff MW, Craig AM (2004) Neurexins induce differentiation of GABA and glutamate postsynaptic specializations via neuroligins. Cell 119:1013-1026.

Hall AC, Lucas FR, Salinas PC (2000) Axonal remodeling and synaptic differentiation in the cerebellum is regulated by WNT-7a signaling. Cell 100:525-535.

Haydon PG, Zoran MJ (1989) Formation and modulation of chemical connections: evoked acetylcholine release from growth cones and neurites of specific identified neurons. Neuron 2:1483-1490.

Henkemeyer M, Itkis OS, Ngo M, Hickmott PW, Ethell IM (2003) Multiple EphB receptor tyrosine kinases shape dendritic spines in the hippocampus. J Cell Biol 163:1313-1326.

Hsueh YP, Sheng M (1999) Regulated expression and subcellular localization of syndecan heparan sulfate proteoglycans and the syndecan-binding protein CASK/LIN-2 during rat brain development. J Neurosci 19:7415-7425.

Hsueh YP, Yang FC, Kharazia V, Naisbitt S, Cohen AR, Weinberg RJ, Sheng M (1998) Direct interaction of CASK/LIN-2 and syndecan heparan sulfate proteoglycan and their overlapping distribution in neuronal synapses. J Cell Biol 142:139-151.

Jacobson M, Baker RE (1968) Neuronal specification of cutaneous nerves through connections with skin grafts. Science 160:543-545.

Jin Y, Garner CC (2008) Molecular mechanisms of presynaptic differentiation. Annu Rev Cell Dev Biol 24:237-262.

Johnson KG, Tenney AP, Ghose A, Duckworth AM, Higashi ME, Parfitt K, Marcu O, Heslip TR, Marsh JL, Schwarz TL, Flanagan JG, Van Vactor D (2006) The HSPGs syndecan and dallylike bind the receptor phosphatase LAR and exert distinct effects on synaptic development. Neuron 49:517-531.

Jontes JD, Phillips GR (2006) Selective stabilization and synaptic specificity: a new cell-biological model. Trends Neurosci 29:186-191.

Jontes JD, Buchanan J, Smith SJ (2000) Growth cone and dendrite dynamics in zebrafish embryos: early events in synaptogenesis imaged in vivo. Nat Neurosci 3:231-237.

Katz LC, Shatz CJ (1996) Synaptic activity and the construction of cortical circuits. Science 274:1133-1138.

Kayser MS, McClelland AC, Hughes EG, Dalva MB (2006) Intracellular and trans-synaptic regulation of glutamatergic synaptogenesis by EphB receptors. J Neurosci 26:12152-12164.

Kim E, Sheng M (2004) PDZ domain proteins of synapses. Nat Rev Neurosci 5:771-781.

Kim S, Burette A, Chung HS, Kwon SK, Woo J, Lee HW, Kim K, Kim H, Weinberg RJ, Kim E (2006) NGL family PSD-95-interacting adhesion molecules regulate excitatory synapse formation. Nat Neurosci 9:12941301.

Kleene R, Schachner M (2004) Glycans and neural cell interactions. Nat Rev Neurosci 5:195-208.

Ko J, Kim S, Chung HS, Kim K, Han K, Kim H, Jun H, Kaang BK, Kim E (2006) SALM Synaptic cell adhesion-like molecules regulate the differentiation of excitatory synapses. Neuron 50:233-245.

Krueger SR, Kolar A, Fitzsimonds RM (2003) The presynaptic release apparatus is functional in the absence of dendritic contact and highly mobile within isolated axons. Neuron 40:945-957.

Kwiatkowski AV, Rubinson DA, Dent EW, Edward van Veen J, Leslie JD, Zhang J, Mebane LM, Philippar U, Pinheiro EM, Burds AA, Bronson RT, Mori S, Fässler R, Gertler FB (2007) Ena/VASP is required for neuritogenesis in the developing cortex. Neuron 56:441-455.

Kwiatkowski AV, Garner CC, Nelson WJ, Gertler FB (2009) Cell autonomous defects in cortical development revealed by two-color chimera analysis. Mol Cell Neurosci 41:44-50.

Lauri SE, Kaukinen S, Kinnunen T, Ylinen A, Imai S, Kaila K, Taira T, Rauvala H (1999) Regulatory role and molecular interactions of a cell-surface heparan sulfate proteoglycan ( $N$-syndecan) in hippocampal long-term potentiation. J Neurosci 19:1226-1235.

Leal-Ortiz S, Waites CL, Terry-Lorenzo R, Zamorano P, Gundelfinger ED, Garner CC (2008) Piccolo modulation of Synapsinla dynamics regulates synaptic vesicle exocytosis. J Cell Biol 181:831-846.

Ledda F, Paratcha G, Sandoval-Guzmán T, Ibáñez CF (2007) GDNF and GFR $\alpha 1$ promote formation of neuronal synapses by ligand-induced cell adhesion. Nat Neurosci 10:293-300.

Li Q, Lau A, Morris TJ, Guo L, Fordyce CB, Stanley EF (2004) A syntaxin 1, $\mathrm{G} \alpha \mathrm{o}$, and $\mathrm{N}$-type calcium channel complex at a presynaptic nerve terminal: analysis by quantitative immunocolocalization. J Neurosci 24:4070-4081.

Matsumoto Y, Irie F, Inatani M, Tessier-Lavigne M, Yamaguchi Y (2007) Netrin-1/DCC signaling in commissural axon guidance requires cellautonomous expression of heparan sulfate. J Neurosci 27:4342-4350.

Mislick KA, Baldeschwieler JD (1996) Evidence for a role of proteoglycans in cation-mediated gene transfer. Proc Natl Acad Sci USA 93:12349-12354.

Missler M, Zhang W, Rohlmann A, Kattenstroth G, Hammer RE, Gottmann K, Südhof TC (2003) $\alpha$-Neurexins couple $\mathrm{Ca}^{2+}$ channels to synaptic vesicle exocytosis. Nature 423:939-948.

Nakase I, Tadokoro A, Kawabata N, Takeuchi T, Katoh H, Hiramoto K, Negishi M, Nomizu M, Sugiura Y, Futaki S (2007) Interaction of arginine-rich peptides with membrane-associated proteoglycans is crucial for induction of actin reorganization and macropinocytosis. Biochemistry 46:492-501.

Noakes PG, Gautam M, Mudd J, Sanes JR, Merlie JP (1995) Aberrant differentiation of neuromuscular junctions in mice lacking s-laminin/laminin [beta]2. Nature 374:258-262.

Okabe S (2007) Molecular anatomy of the postsynaptic density. Mol Cell Neurosci 34:503-518.

Okabe S, Miwa A, Okado H (2001) Spine formation and correlated assembly of presynaptic and postsynaptic molecules. J Neurosci 21:6105-6114.

Peng HB, Markey DR, Muhlach WL, Pollack ED (1987) Development of presynaptic specializations induced by basic polypeptide-coated latex beads in spinal cord cultures. Synapse 1:10-19.

Phillips GR, Huang JK, Wang Y, Tanaka H, Shapiro L, Zhang W, Shan WS, Arndt K, Frank M, Gordon RE, Gawinowicz MA, Zhao Y, Colman DR (2001) The presynaptic particle web: ultrastructure, composition, dissolution and reconstitution. Neuron 32:63-77.

Rauvala H, Peng HB (1997) HB-GAM (Heparin-binding growth-associated molecule) and heparin-type glycans in the development and plasticity of neuron-target contacts. Prog Neurobiol 52:127-144.

Ryan TA, Reuter H, Wendland B, Schweizer FE, Tsien RW, Smith SJ (1993) The kinetics of synaptic vesicle recycling measured at single presynaptic boutons. Neuron 11:713-724.

Sankaranarayanan S, Atluri PP, Ryan TA (2003) Actin has a molecular scaf- 
folding, not propulsive, role in presynaptic function. Nat Neurosci 6:127-135.

Sara Y, Biederer T, Atasoy D, Chubykin A, Mozhayeva MG, Südhof TC, Kavalali ET (2005) Selective capability of synCAM and neuroligin for functional synapse assembly. J Neurosci 25:260-270.

Sato S, Omori Y, Katoh K, Kondo M, Kanagawa M, Miyata K, Funabiki K, Koyasu T, Kajimura N, Miyoshi T, Sawai H, Kobayashi K, Tani A, Toda T, Usukura J, Tano Y, Fujikado T, Furukawa T (2008) Pikachurin, a dystroglycan ligand, is essential for photoreceptor ribbon synapse formation. Nat Neurosci 11:923-931.

Schacher S, Rayport SG, Ambron RT (1985) Giant Aplysia neuron R2 reliably forms strong chemical connections in vitro. J Neurosci 5:2851-2856.

Scheiffele P, Fan J, Choih J, Fetter R, Serafini T (2000) Neuroligin expressed in nonneuronal cells triggers presynaptic development in contacting axons. Cell 101:657-669.

Schlüter OM, Xu W, Malenka RC (2006) Alternative N-terminal domains of PSD-95 and SAP97 govern activity-dependent regulation of synaptic AMPA receptor function. Neuron 51:99-111.

Shapira M, Zhai RG, Dresbach T, Bresler T, Torres VI, Gundelfinger ED, Ziv NE, Garner CC (2003) Unitary assembly of presynaptic active zones from piccolo-bassoon transport vesicles. Neuron 38:237-252.

Shapiro L, Love J, Colman DR (2007) Adhesion molecules in the nervous system: Structural insights into function and diversity. Annu Rev Neurosci 30:451-474.

Spector I, Braet F, Shochet NR, Bubb MR (1999) New anti-actin drugs in the study of the organization and function of the actin cytoskeleton. Microsc Res Tech 47:18-37.

Sperry RW (1963) Chemoaffinity in the orderly growth of nerve fiber patterns and connections. Proc Natl Acad Sci U S A 50:703-710.

Sugiura M, Dow KE (1994) Activity-dependent regulation of neuronal synthesis and release of neurite-promoting heparan sulfate proteoglycans. Dev Biol 164:102-110.

Sur M, Garraghty PE, Roe AW (1988) Experimentally induced visual projections into auditory thalamus and cortex. Science 242:1437-1441.

Tsuriel S, Geva R, Zamorano P, Dresbach T, Boeckers T, Gundelfinger ED, Garner CC, Ziv NE (2006) Local sharing as a predominant determinant of synaptic matrix molecular dynamics. PLoS Biol 4:e271.
Ullian EM, Sapperstein SK, Christopherson KS, Barres BA (2001) Control of synapse number by glia. Science 291:657-661.

Umemori H, Linhoff MW, Ornitz DM, Sanes JR (2004) FGF22 and its close relatives are presynaptic organizing molecules in the mammalian brain Cell 118:257-270.

van der Weyden L, Arends MJ, Chausiaux OE, Ellis PJ, Lange UC, Surani MA, Affara N, Murakami Y, Adams DJ, Bradley A (2006) Loss of TSLC1 causes male infertility due to a defect at the spermatid stage of spermatogenesis. Mol Cell Biol 26:3595-3609.

Van Vactor D, Wall DP, Johnson KG (2006) Heparan sulfate proteoglycans and the emergence of neuronal connectivity. Curr Opin Neurobiol 16:40-51.

Varoqueaux F, Aramuni G, Rawson RL, Mohrmann R, Missler M, Gottmann K, Zhang W, Südhof TC, Brose N (2006) Neuroligins determine synapse maturation and function. Neuron 51:741-754.

Waites CL, Specht CG, Härtel K, Leal-Ortiz S, Genoux D, Li D, Drisdel RC Jeyifous O, Cheyne JE, Green WN, Montgomery JM, Garner CC (2009) Synaptic SAP97 isoforms regulate AMPA receptor dynamics and access to presynaptic glutamate. J Neurosci 29:4332-4345.

Webb SJ, Monk CS, Nelson CA (2001) Mechanisms of postnatal neurobiological development: implications for human development. Dev Neuropsychol 19:147-171.

Withers GS, Higgins D, Charette M, Banker G (2000) Bone morphogenetic protein-7 enhances dendritic growth and receptivity to innervation in cultured hippocampal neurons. Eur J Neurosci 12:106-116.

Yavin E, Yavin Z (1974) Attachment and culture of dissociated cells from rat embryo cerebral hemispheres on polylysine-coated surface. J Cell Biol 62:540-546.

Zhang W, Benson DL (2001) Stages of synapse development defined by dependence on F-actin. J Neurosci 21:5169-5181.

Zhang ZW (2006) Postnatal development of the mammalian neocortex: role of activity revisited. Can J Neurol Sci 33:158-169.

Zwimpfer TJ, Aguayo AJ, Bray GM (1992) Synapse formation and preferential distribution in the granule cell layer by regenerating retinal ganglion cell axons guided to the cerebellum of adult hamsters. J Neurosci 12:1144-1159. 